MATHEMATICS OF COMPUTATION

Volume 71, Number 240, Pages 1371-1403

S 0025-5718(01)01401-6

Article electronically published on December 4, 2001

\title{
ERROR INDICATORS \\ FOR THE MORTAR FINITE ELEMENT DISCRETIZATION OF THE LAPLACE EQUATION
}

\author{
CHRISTINE BERNARDI AND FRÉDÉRIC HECHT
}

\begin{abstract}
The mortar technique turns out to be well adapted to handle mesh adaptivity in finite elements, since it allows for working with nonnecessarily compatible discretizations on the elements of a nonconforming partition of the initial domain. The aim of this paper is to extend the numerical analysis of residual error indicators to this type of methods for a model problem and to check their efficiency thanks to some numerical experiments.
\end{abstract}

\section{INTRODUCTION}

The mortar element method [6], [7] is a domain decomposition technique which allows for working with completely independent discretizations on the subdomains of a partition of the domain without overlapping. So it seems ideally suited for mesh adaptivity. Indeed, nonmatching grids can be used on the different subdomains of a partition, and this leads to highly reducing the number of degrees of freedom in order to satisfy the adaptivity criteria since no further node must be added for conformity reasons. Also, the regularity properties of the initial grid are preserved.

The a priori analysis of the corresponding discrete problem - note that the mortar method is most often nonconforming - has already been performed for the Laplace equation in the case of finite element discretizations [5] or spectral discretizations [1] and also for the Stokes problem [3. However, the a posteriori analysis still seems unsufficient for this method. We refer to [10] and [19] for the extension of residual type error indicators to the case of nonconforming finite elements but on conforming triangulations, and also to [8] and [23] for first estimates concerning these indicators in the mortar framework. However, in most of these papers saturation assumptions are made, and we think that they could be avoided in a large number of cases.

The aim of this paper is to extend the estimates concerning the residual type indicators to the mortar finite element discretization of a model problem, more precisely of the Laplace equation in a polygon $\Omega$

$$
\begin{cases}-\Delta u=f & \text { in } \Omega, \\ u=0 & \text { on } \partial \Omega .\end{cases}
$$

Received by the editor April 4, 2000 and, in revised form, October 10, 2000

2000 Mathematics Subject Classification. Primary 65N30; Secondary 65N50, 65N55.

(C)2001 American Mathematical Society 
We first explain how mesh adaptivity can be performed in this case and present the corresponding discrete problems. Relying on [5] for some basic results, we perform the a priori analysis of these problems. Next, we introduce the residual error indicators related to the discretization. In contrast to most standard definitions (see 20 and the references therein) and as already suggested in 10, two kinds of indicators are defined: the first ones are associated with all elements of the triangulation and deal with the finite element discretization, the second ones are associated with the edges contained in the skeleton of the partition and deal with the nonconformity of the discretization. The main results of this paper consist in deriving estimates which allow for comparing them with the error. These results do not require any saturation assumption and they are optimal, in the sense that the constants involved in the estimates are independent of the discretization parameter. We conclude with some numerical experiments which turn out to be in good coherency with the analysis.

An outline of the paper is as follows:

- In Section 2, we describe the successive refined meshes, together with the corresponding discrete spaces and problems. We prove their well-posedness together with some a priori error estimates.

- In Section 3, we introduce the two kinds of residual type error indicators. Next, we perform the a posteriori analysis, by proving upper bounds first for the error, second for the indicators.

- Section 4 is devoted to some numerical tests of adaptivity.

- A technical proof is given in an Appendix.

\section{The REFINED MEShes AND THE DISCRETE PROBlEMS}

Let $\Omega$ be a connected and bounded open set in $\mathbb{R}^{2}$, with a Lipschitz-continuous boundary. In order to avoid curved elements, we assume that $\Omega$ is a polygon.

2.a. The family of refined triangulations. Let $\left(\mathcal{T}_{h}^{0}\right)_{h^{0}}$ be a family of "coarse" triangulations of the domain $\Omega$ in the usual sense: each $\mathcal{T}_{h}^{0}$ is a finite set of triangles such that $\bar{\Omega}$ is the union of these triangles and the intersection of two different elements of $\mathcal{T}_{h}^{0}$, if not empty, is a vertex or a whole edge of both of them. As usual, $h^{0}$ denotes the maximal diameter of the elements of $\mathcal{T}_{h}^{0}$. We make the further assumption that this family is regular, i.e., there exists a positive constant $\sigma$ such that, for all $h^{0}$ and for all $K$ in $\mathcal{T}_{h}^{0}$, the ratio of the diameter of $K$ to the diameter of its inscribed circle is smaller than $\sigma$.

Starting from this family $\left(\mathcal{T}_{h}^{0}\right)_{h^{0}}$, we build iteratively new families of refined triangulations as follows. Assuming that the family $\left(\mathcal{T}_{h}^{n-1}\right)_{h^{n-1}}$ is known, for each value of the parameter $h^{n-1}$ :

- for arbitrary positive integers $\ell$, we cut some elements of $\mathcal{T}_{h}^{n-1}$ into $2^{2 \ell}$ subtriangles by iteratively joining the midpoints of the edges of these elements;

- we denote by $\mathcal{T}_{h}^{n, k}$ the set of triangles which have area equal to $2^{-2 k}$ the area of the triangle $K$ of $\mathcal{T}_{h}^{0}$ in which they are contained;

- we denote by $\Omega^{n, k}$ the open subdomain of $\Omega$ such that $\bar{\Omega}^{n, k}$ is the union of the triangles of $\mathcal{T}_{h}^{n, k}$;

- we call $\mathcal{T}_{h}^{n}$ the union of the $\mathcal{T}_{h}^{n, k}$.

Figure 1 presents an example of triangulation $\mathcal{T}_{h}^{n}$. 


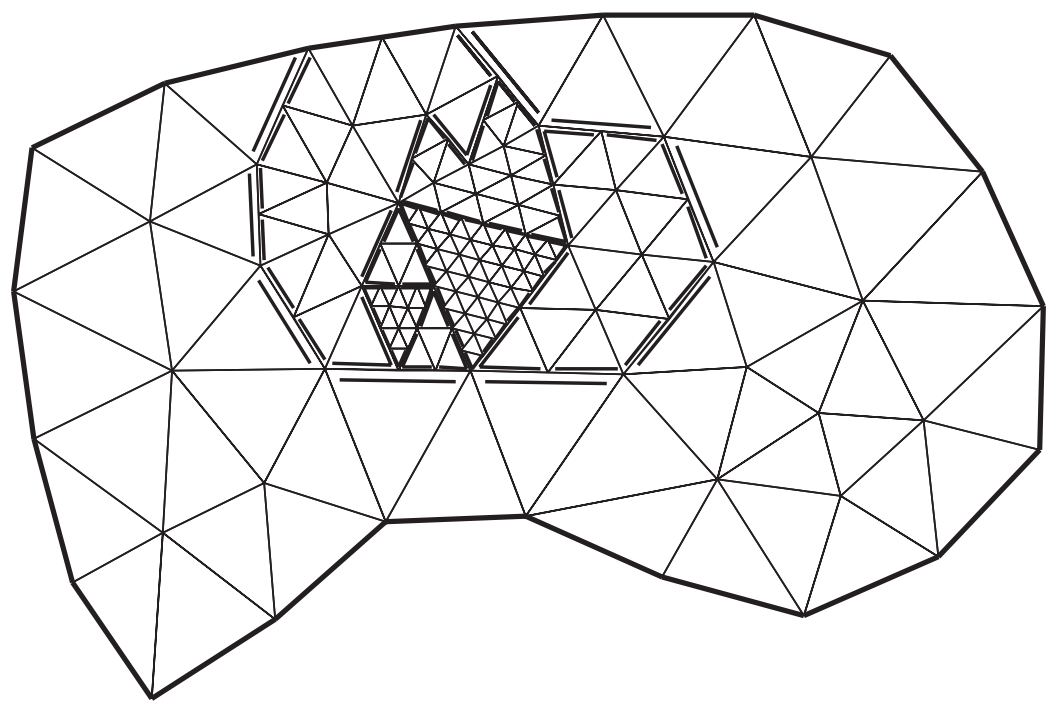

Figure 1.

Clearly, for each $n$, there exists a nonnegative integer $K^{n}$ such that

$$
\bar{\Omega}=\bigcup_{k=0}^{K^{n}} \bar{\Omega}^{n, k} \quad \text { and } \quad \Omega^{n, k} \cap \Omega^{n, k^{\prime}}=\emptyset, \quad 0 \leq k<k^{\prime} \leq K^{n},
$$

which means that for each $n$ the $\Omega^{n, k}, 0 \leq k \leq K^{n}$, form a partition of the domain $\Omega$ without overlapping. Moreover, the parameters $h^{n, k}$ and $h^{n}$ are defined in an obvious way as the maximal diameters of the triangles of $\mathcal{T}_{h}^{n, k}$ and $\mathcal{T}_{h}^{n}$, respectively. So they satisfy

$$
h^{n, k} \leq 2^{-k} h^{0} \quad \text { and } \quad h^{n}=\max _{0 \leq k \leq K^{n}} h^{n, k} .
$$

Remark. Even if the technique we propose for refining the mesh is rather general, it can be extended in several ways, for instance by cutting the triangles into $(m+1)^{2 \ell}$ subelements for any nonnegative integer $m$ instead of $2^{2 \ell}$ or starting from an a priori decomposition of the domain $\Omega$ and using independent family of triangulations on each subdomain. It can also be extended to three-dimensional triangulations into tetrahedra if a technique for cutting a tetrahedron is fixed. Moreover, coarsening the mesh is also easy to perform by keeping in memory the previous triangulation and going back to it where necessary.

To conclude, at each step $n$, we define the skeleton

$$
\mathcal{S}^{n}=\bigcup_{k=0}^{K^{n}} \partial \Omega^{n, k} \backslash \partial \Omega
$$

and, as standard in the mortar method [6], we fix a decomposition of it into disjoint (open) mortars

$$
\overline{\mathcal{S}}^{n}=\bigcup_{m=1}^{M^{n}} \bar{\gamma}^{m} \quad \text { and } \quad \gamma^{m} \cap \gamma^{m^{\prime}}=\emptyset, \quad 1 \leq m<m^{\prime} \leq M^{n} .
$$




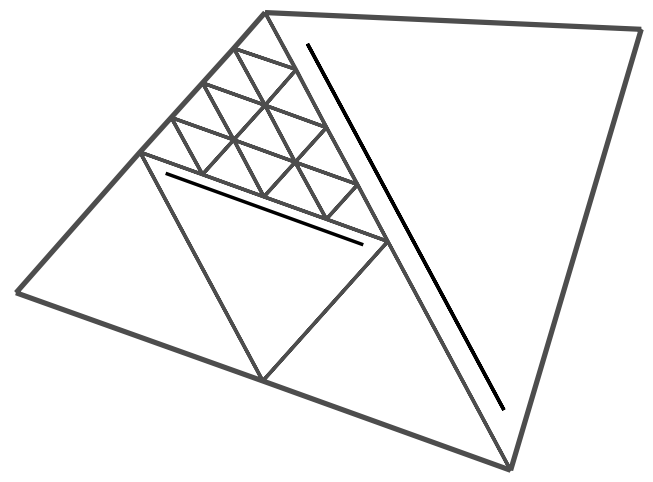

FIGURE 2.

We make the final assumption that each $\bar{\gamma}^{m}, 1 \leq m \leq M^{n}$, is a whole edge of a triangle of one of the triangulations $\mathcal{T}_{h}^{n, k}$, located on one side of $\gamma^{m}$, and that on the other side it is the union of edges of triangles in $\mathcal{T}_{h}^{n, k_{1}} \cup \cdots \cup \mathcal{T}_{h}^{n, k_{p}}$, where all $k_{i}$ are $>k$. We agree to denote by $k(m), k_{1}(m), \ldots, k_{p}(m)$, the corresponding exponents $k, k_{1}, \ldots, k_{p}$, and by $p(m)$ the number $p$. This is illustrated in Figure 2, with two mortars and $p(m)$ equal to 1 and 2 , respectively.

2.b. The mortar discrete spaces. The discretization parameter $\delta$ is now the pair $\left(n, h^{n}\right)$. We fix a positive integer $\ell$ and, with each value of $\delta$, we associate the local discrete spaces, for $0 \leq k \leq K^{n}$,

$$
X^{n, k}=\left\{v_{k} \in \mathcal{C}^{0}\left(\bar{\Omega}^{n, k}\right) ; \forall K \in \mathcal{T}_{h}^{n, k}, v_{k \mid K} \in \mathcal{P}_{\ell}(K)\right\},
$$

where $\mathcal{P}_{\ell}(K)$ stands for the space of restrictions to $K$ of polynomials with total degree $\leq \ell$.

Now let $\gamma^{m}, 1 \leq m \leq M^{n}$, be one of the mortars. Then, two possible choices exist for defining the discrete space on $\gamma^{m}$ which is used to enforce the matching conditions. With the previous notation:

- The coarse space $\widetilde{W}_{\mathrm{C}}^{m}$ is the space $\mathcal{P}_{\ell-1}\left(\gamma^{m}\right)$ of polynomials with degree $\leq \ell-1$ on $\gamma^{m}$. In this case, we agree to denote by $\mathcal{E}^{m}$ the set $\left\{\gamma^{m}\right\}$.

- Otherwise, we define $\mathcal{E}^{m}$ as the set of the open connected components of the intersections $\bar{\gamma}^{m} \cap \partial \Omega^{n, k_{i}(m)}, 1 \leq i \leq p(m)$. The fine space $\widetilde{W}_{\mathrm{F}}^{m}$ is chosen as

$$
\widetilde{W}_{\mathrm{F}}^{m}=\left\{\varphi_{\delta} \in L^{2}\left(\gamma^{m}\right) ; \forall e \in \mathcal{E}^{m}, \varphi_{\mid e} \in \widetilde{W}^{m}(e)\right\},
$$

where each $\widetilde{W}^{m}(e)$ is the space of continuous functions on $e$ such that their restrictions to each edge $e^{\prime}=e \cap \partial K$ for all $K$ in $\mathcal{T}_{h}^{n, k_{i}}$ belongs to $\mathcal{P}_{\ell-1}\left(e^{\prime}\right)$ if $e^{\prime}$ contains an endpoint of $e$, to $\mathcal{P}_{\ell}\left(e^{\prime}\right)$ if not.

Note that these choices are rather standard in the mortar framework [6].

The discrete space $\mathbb{X}_{\delta}$ is now defined in the usual way (see [7]). It is the space of functions $v_{\delta}$ such that:

- their restrictions to each $\Omega^{n, k}, 0 \leq k \leq K^{n}$, belong to $X^{n, k}$;

- they vanish on $\partial \Omega$; 
- the following matching condition holds on any $\gamma^{m}, 1 \leq m \leq M^{n}$,

$$
\forall \chi \in \widetilde{W}^{m}, \quad \int_{\gamma^{m}}\left[v_{\delta}\right](\tau) \chi(\tau) d \tau=0,
$$

where $\widetilde{W}^{m}$ is one of the two spaces $\widetilde{W}_{\mathrm{C}}^{m}$ or $\widetilde{W}_{\mathrm{F}}^{m}$.

Remark. As proposed in the first version of the mortar method [6], some further matching conditions can be added; more precisely, the functions in $\mathbb{X}_{\delta}$ can be enforced to be continuous at the endpoints of all $\gamma^{m}$. These conditions are satisfied in the numerical experiments of this paper, but they are not necessary for the a priori analysis.

Note that the choice of $\widetilde{W}^{m}$ is local, i.e., independent choices of $\widetilde{W}_{\mathrm{C}}^{m}$ or $\widetilde{W}_{\mathrm{F}}^{m}$ are possible according to the mortars. Each choice $\widetilde{W}_{\mathrm{C}}^{m}$ results in $\ell$ matching conditions, while each choice $\widetilde{W}_{\mathrm{F}}^{m}$ results in at least $2 \ell-1$ and often more matching conditions. However, in this case, the matching condition (2.6) can be enforced separately on each $e$ in $\mathcal{E}^{m}$ and the jump here is equal to $v_{\delta \mid \Omega^{n, k(m)}}-v_{\delta \mid \Omega^{n, k_{i}(m)}}$, hence only requires the knowledge of $v_{\delta}$ on two subdomains.

Remark. The local spaces $X^{n, k}$ are conforming in the sense that they are contained in $H^{1}\left(\Omega^{n, k}\right)$ and, moreover, the nullity conditions on $\partial \Omega$ are exactly taken into account in $\mathbb{X}_{\delta}$. However, the global space $\mathbb{X}_{\delta}$ is not contained in $H^{1}(\Omega)$, except for some very special geometries of the decomposition and when all the $\widetilde{W}^{m}$ are chosen equal to $\widetilde{W}_{\mathrm{F}}^{m}$.

2.c. The discrete problems. The discrete problems associated with equation (1.1) are now built from its variational formulation by the Galerkin method. For fixed data $f$ in $L^{2}(\Omega)$, they read as follows:

Find $u_{\delta}$ in $\mathbb{X}_{\delta}$ such that

$$
\forall v_{\delta} \in \mathbb{X}_{\delta}, \quad a_{\delta}\left(u_{\delta}, v_{\delta}\right)=\int_{\Omega} f(\boldsymbol{x}) v_{\delta}(\boldsymbol{x}) d \boldsymbol{x},
$$

where the bilinear form $a_{\delta}(\cdot, \cdot)$ is defined by

$$
a_{\delta}\left(u_{\delta}, v_{\delta}\right)=\sum_{k=0}^{K^{n}} \int_{\Omega^{n, k}} \operatorname{grad} u_{\delta} \cdot \operatorname{grad} v_{\delta} d \boldsymbol{x} .
$$

We must now check the well-posedness of problem (2.7). Since the space $\mathbb{X}_{\delta}$ is not contained in $H^{1}(\Omega)$ in the general case, we introduce the decomposition-dependent norm, indexed by $\delta$ :

$$
\|v\|_{H_{\delta}^{1}(\Omega)}=\left(\sum_{k=0}^{K^{n}}\|v\|_{H^{1}\left(\Omega^{n, k}\right)}^{2}\right)^{\frac{1}{2}} .
$$

The form $a_{\delta}(\cdot, \cdot)$ is obviously continuous with respect to this norm, and its ellipticity can be checked by exactly the same arguments as in [5, Prop. 2.1] where only two families of triangulations are considered (another proof, relying on a duality argument, is due to [14]). So we state the result without proof, in a slightly more general form which is needed later on. It requires the following assumption.

Assumption A.1. For $1 \leq m \leq M^{n}$, either the space $\widetilde{W}^{m}$ coincides with $\widetilde{W}_{\mathrm{F}}^{m}$ or $p(m)$ is equal to 1 . 
Lemma 2.1. If Assumption A.1 is satisfied, there exists a constant $\alpha$ only depending on the geometry of $\Omega$ and the parameter $\sigma$ that measures the regularity of the family $\left(\mathcal{T}_{h}^{0}\right)_{h^{0}}$ such that the following ellipticity property holds for any value of the parameter $\delta$ :

$$
\forall v \in \mathbb{X}^{n}, \quad a_{\delta}(v, v) \geq \alpha\|v\|_{H_{\delta}^{1}(\Omega)}^{2},
$$

where $\mathbb{X}^{n}$ is the space of functions $v$ such that

- their restrictions to each $\Omega^{n, k}, 0 \leq k \leq K^{n}$, belong to $H^{1}\left(\Omega^{n, k}\right)$,

- they vanish on $\partial \Omega$,

- the following matching condition holds on any $\gamma^{m}, 1 \leq m \leq M^{n}$,

$$
\forall e \in \mathcal{E}^{m}, \quad \int_{e}[v](\tau) d \tau=0
$$

Since $\mathbb{X}_{\delta}$ is contained in $\mathbb{X}^{n}$ for all $\delta$ (this follows from its definition), using this lemma leads to the well-posedness and stability properties of the problem.

Corollary 2.2. If Assumption A.1 is satisfied, for any data $f$ in $L^{2}(\Omega)$ and for any value of the parameter $\delta$, problem (2.7) has a unique solution. Moreover, this solution satisfies, for a constant $c$ independent of $\delta$,

$$
\left\|u_{\delta}\right\|_{H_{\delta}^{1}(\Omega)} \leq c\|f\|_{L^{2}(\Omega)} .
$$

2.d. A priori analysis. The numerical analysis of problem (2.7) has been performed in [5] in the simpler case of one coarse and one fine triangulations; however, most results extend to the present case. So we briefly recall them and make precise the evaluation of the approximation error, which is more technical in our framework.

If Assumption A.1 holds, we derive from Lemma 2.1 the following Strang's lemma

$$
\left\|u-u_{\delta}\right\|_{H_{\delta}^{1}(\Omega)} \leq\left(1+\alpha^{-1}\right) \inf _{v_{\delta} \in \mathbb{X}_{\delta}}\left\|u-v_{\delta}\right\|_{H_{\delta}^{1}(\Omega)}+\alpha^{-1} \sup _{w_{\delta} \in \mathbb{X}_{\delta}} \frac{\int_{\mathcal{S}^{n}} \partial_{n} u\left[w_{\delta}\right] d \tau}{\left\|w_{\delta}\right\|_{H_{\delta}^{1}(\Omega)}},
$$

where $\partial_{n}$ denotes the normal derivative on $\Gamma$ and $[\cdot]$ the jump through $\mathcal{S}^{n}$ with the appropriate sign. It can be observed that, in the right-hand side of (2.12),

- the first term represents the approximation error,

- the second term, due to the nonconformity of the discretization, represents the consistency error.

Evaluating the consistency error relies on the matching condition (2.6), which implies for all $m, 1 \leq m \leq M^{n}$,

$$
\forall \chi \in \widetilde{W}_{\mathrm{C}}^{m} \subset \widetilde{W}^{m}, \quad \int_{\gamma^{m}} \partial_{n} u\left[w_{\delta}\right] d \tau=\int_{\gamma^{m}}\left(\partial_{n} u-\chi\right)\left[w_{\delta}\right] d \tau .
$$

Hence, by the the same arguments as in [5. Prop. 2.4], we derive, with the appropriate smoothness assumptions on $u$ and for $s_{k} \leq \ell+1$,

$$
\sup _{w_{\delta} \in \mathbb{X}_{\delta}} \frac{\int_{\mathcal{S}^{n}} \partial_{n} u\left[w_{\delta}\right] d \tau}{\left\|w_{\delta}\right\|_{H_{\delta}^{1}(\Omega)}} \leq c\left(\sum_{k=0}^{K^{n}}\left(h^{n, k}\right)^{2\left(s_{k}-1\right)}\|u\|_{H^{s_{k}\left(\Omega^{n, k}\right)}}^{2}\right)^{\frac{1}{2}}
$$

for a constant $c$ independent of $\delta$. 


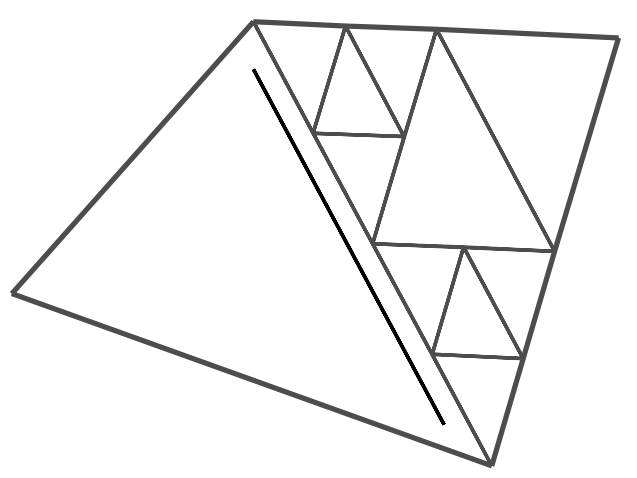

FIGURE 3.

We now evaluate the approximation error, which is much more complex. We introduce the following parameter, which is equal to the maximal ratio of the diameters of two adjacent triangles:

$$
\mu_{\delta}=\sup _{1 \leq m \leq M^{n}} \sup _{1 \leq i \leq p(m)} 2^{k_{i}(m)-k(m)} .
$$

For each $m$, the intersection of $\gamma^{m}$ and $\partial \Omega^{n, k_{i}(m)}, 1 \leq i \leq p(m)$, has a finite number of (open) connected components, which we denote by $\gamma_{i j}^{m}, 1 \leq j \leq q(i)$ (this is illustrated in Figure 3, where $q(i)$ is equal to 2). Let $\Delta^{k(m)}$ denote the triangle of $\mathcal{T}_{h}^{n, k(m)}$ such that $\gamma^{m}$ is an edge of $\Delta^{k(m)}$ and $\Delta_{j}^{k_{i}(m)}$ denote the union of triangles in $\mathcal{T}_{h}^{n, k_{i}(m)}$ that intersect $\gamma_{i j}^{m}$. Lemma 2.3 requires the existence of a lifting operator $L_{i j}^{m}$, from the space of traces on $\gamma_{i j}^{m}$ of functions in $X^{n, k_{i}(m)}$ vanishing at the endpoints of $\gamma_{i j}^{m}$, with values in $X^{n, k_{i}(m)}$, such that, for such a trace $\varphi, L_{i j}^{m} \varphi$ vanishes on $\partial \Delta_{j}^{k_{i}(m)} \backslash \gamma^{m}$ and satisfies

$$
\left\|L_{i j}^{m} \varphi\right\|_{H^{1}\left(\Delta_{j}^{k_{i}(m)}\right)} \leq c \mu_{\delta}^{\frac{1}{2}}|\varphi|_{H_{00}^{\frac{1}{2}\left(\gamma_{i j}^{m}\right)}},
$$

where, as standard, $H_{00}^{\frac{1}{2}}\left(\gamma_{i j}^{m}\right)$ denotes the interpolate of order $\frac{1}{2}$ between $H_{0}^{1}\left(\gamma_{i j}^{m}\right)$ and $L^{2}\left(\gamma_{i j}^{m}\right)$ (see [17, Chap. 1, Th. 11.7]). Such an operator is constructed in [4] form. (5.1)], according to an idea of [22, on a fixed polygon by combining the use of Clément's regularization operator [13] with a "continuous" lifting operator from $H_{00}^{\frac{1}{2}}\left(\gamma_{i j}^{m}\right)$ into $H^{1}\left(\Delta_{j}^{k_{i}(m)}\right)$. The extension to domains $\Delta_{j}^{k_{i}(m)}$ when taking into account their aspect ratios (which are bounded by $\mu_{\delta}$ ) can be performed as in [ [5. Cor. 3.6] by going to a reference trapezium.

Lemma 2.3. Let us assume the solution u of problem (1.1) such that, for all $n$, each $u_{\mid \Omega^{n, k}}, 0 \leq k \leq K^{n}$, belongs to $H^{s_{k}}\left(\Omega^{n, k}\right), 1<s_{k} \leq \ell+1$. There exists a constant $c$ independent of $\delta$ such that

$$
\inf _{v_{\delta} \in \mathbb{X}_{\delta}}\left\|u-v_{\delta}\right\|_{H_{\delta}^{1}(\Omega)} \leq c \mu_{\delta}^{\frac{1}{2}}\left(\sum_{k=0}^{K^{n}}\left(h^{n, k}\right)^{2\left(s_{k}-1\right)}\|u\|_{H^{s_{k}\left(\Omega^{n, k}\right)}}^{2}\right)^{\frac{1}{2}} .
$$

Proof. The construction of an appropriate $v_{\delta}$ is performed in two steps.

1) With each $\Omega^{n, k}$, we associate the set $\mathcal{V}^{n, k}$ of its corners $\boldsymbol{a}$ which are inside a $\gamma^{m}$ (then $\Omega^{n, k}$ coincides with an $\Omega^{n, k_{i}(m)}$ ). For each $\boldsymbol{a}$ in $\mathcal{V}^{n, k}$, we consider the 
Lagrange function $\varphi_{\boldsymbol{a}}$ in $X^{n, k}$ associated with $\boldsymbol{a}$. If $\mathcal{I}^{n, k}$ denotes the standard Lagrange interpolation operator with values in $X^{n, k}$, the idea is to define a first function $v_{\delta}^{1}$ by

$$
\left(v_{\delta}^{1}\right)_{\mid \Omega^{n, k}}=\mathcal{I}^{n, k} u+\sum_{\boldsymbol{a} \in \mathcal{V}^{n, k}}\left[\mathcal{I}_{\delta} u\right](\boldsymbol{a}) \varphi_{\boldsymbol{a}}, \quad 0 \leq k \leq K^{n}
$$

where, if $\Omega^{n, k}$ coincides with $\Omega^{n, k_{i}(m)}$, the jump $\left[\mathcal{I}_{\delta} u\right](\boldsymbol{a})$ means $\mathcal{I}^{n, k(m)} u-\mathcal{I}^{n, k_{i}(m)} u$. Standard results [12, Thm. 16.2] yield that

$$
\left\|u-\mathcal{I}^{n, k} u\right\|_{H^{1}\left(\Omega^{n, k}\right)} \leq c\left(h^{n, k}\right)^{s_{k}-1}\|u\|_{H^{s_{k}\left(\Omega^{n, k}\right)}} .
$$

Also it is readily checked by going to a reference triangle that $\left\|\varphi_{\boldsymbol{a}}\right\|_{H^{1}\left(\Omega^{n, k}\right)}$ is bounded independently of $\delta$. In order to bound $\left[\mathcal{I}_{\delta} u\right](\boldsymbol{a})$, we introduce a continuous one-to-one mapping $F$ from the union $\bar{\Delta}^{k(m)} \cup \bar{\Delta}_{j}^{k_{i}(m)}$ onto a reference subdomain $\hat{\Delta}$, which is affine on each triangle of $\mathcal{T}_{h}^{n}$ contained in $\bar{\Delta}^{k(m)} \cup \bar{\Delta}_{j}^{k_{i}(m)}$ and maps the edge $e$ of the triangle of $\Delta_{j}^{k_{i}(m)}$ containing $\boldsymbol{a}$ and contained in $\gamma^{m}$ onto an edge $\hat{e}$ with length 1 , the edge $e^{\prime}$ in $\bar{\Delta}^{k(m)}$ that contains $e$ onto an edge $\hat{e}^{\prime}$. Then, if $\hat{w}$ stands for the function $w \circ F^{-1}$ for all functions $w$, we have

$$
\begin{aligned}
\|\left[\mathcal{I}_{\delta} u\right](\boldsymbol{a}) \mid & \leq\left\|\widehat{\mathcal{I}^{n, k(m)}} u-\widehat{\mathcal{I}^{n, k_{i}(m)}} u\right\|_{L^{\infty}(\hat{e})} \leq \hat{c}\left\|\widehat{\mathcal{I}^{n, k(m)}} u-\widehat{\mathcal{I}^{n, k_{i}(m)}} u\right\|_{L^{2}(\hat{e})} \\
& \leq \hat{c}\left(\left\|\hat{u}-\widehat{\mathcal{I}^{n, k(m)}} u\right\|_{L^{2}(\hat{e})}+\left\|\hat{u}-\widehat{\mathcal{I}^{n, k(m)}} u\right\|_{L^{2}\left(\hat{e}^{\prime}\right)}\right) \\
& \leq c\left(\left|u-\mathcal{I}^{n, k(m)} u\right|_{H^{1}\left(\Delta^{k(m)}\right)}+\left|u-\widehat{\mathcal{I}^{n, k(m)}} u\right|_{H^{1}\left(\Delta_{j}^{k_{i}(m)}\right)}\right) .
\end{aligned}
$$

Summing the square of this inequality on all $\boldsymbol{a}$ in $\mathcal{V}^{n, k}$ (the support of a function $\varphi_{a}$ only intersects a finite number of supports of other ones) and applying once more the previous interpolation property leads to

$$
\left\|u-v_{\delta}^{1}\right\|_{H_{\delta}^{1}(\Omega)} \leq c\left(\sum_{k=0}^{K^{n}}\left(h^{n, k}\right)^{2\left(s_{k}-1\right)}\|u\|_{H^{s} k\left(\Omega^{n, k}\right)}^{2}\right)^{\frac{1}{2}} .
$$

2) Since the function $v_{\delta}^{1}$ does not belong to $\mathbb{X}_{\delta}$, the idea consists in adding a correction $v_{\delta}^{2}$ defined by

$$
v_{\delta}^{2}=\sum_{m=1}^{M^{n}} \sum_{i=1}^{p(m)} \sum_{j=1}^{q(i)} L_{i j}^{m}\left(\left(v_{\delta}^{1}\right)_{\mid \Omega^{n, k(m)}}-\left(v_{\delta}^{1}\right)_{\mid \Omega^{n, k_{i}(m)}}\right) .
$$

From (2.15), we have

$$
\left\|v_{\delta}^{2}\right\|_{H_{\delta}^{1}(\Omega)}^{2} \leq c \mu_{\delta} \sum_{m=1}^{M^{n}} \sum_{i=1}^{p(m)} \sum_{j=1}^{q(i)}\left|\left(v_{\delta}^{1}\right)_{\mid \Omega^{n, k(m)}}-\left(v_{\delta}^{1}\right)_{\mid \Omega^{n, k_{i}(m)}}\right|_{H_{00}^{\frac{1}{2}}\left(\gamma_{i j}^{m}\right)}^{2} .
$$

Next, we set

$$
u_{\mid \Omega^{n, k}}^{1}=u_{\mid \Omega^{n, k}}+\sum_{\boldsymbol{a} \in \mathcal{V}^{n, k}}\left[\mathcal{I}_{\delta} u\right](\boldsymbol{a}) \varphi_{\boldsymbol{a}}, \quad 0 \leq k \leq K^{n}
$$

where, as previously, if $\Omega^{n, k}$ coincides with $\Omega^{n, k_{i}(m)}$, the jump $\left[\mathcal{I}_{\delta} u\right](\boldsymbol{a})$ means $\mathcal{I}^{n, k(m)} u-\mathcal{I}^{n, k_{i}(m)} u$. Note that $u$ and $\mathcal{I}^{n, k_{i}(m)} u$ coincide in this point $\boldsymbol{a}$. Hence, 
the function $u^{1}$ coincides with both $\left(v_{\delta}^{1}\right)_{\mid \Omega^{n, k(m)}}$ and $\left(v_{\delta}^{1}\right)_{\mid \Omega^{n, k_{i}(m)}}$ at the endpoints of all $\gamma_{i j}^{m}$, so that

$$
\begin{aligned}
&\left\|v_{\delta}^{2}\right\|_{H_{\delta}^{1}(\Omega)}^{2} \leq c \mu_{\delta} \sum_{m=1}^{M^{n}} \sum_{i=1}^{p(m)} \sum_{j=1}^{q(i)}\left(\mid u^{1}-\left(v_{\delta}^{1}\right)_{\left|\Omega^{n, k(m)}\right|_{H_{00}^{\frac{1}{2}}\left(\gamma_{i j}^{m}\right)}^{2}}\right.\left.+\mid u^{1}-\left(v_{\delta}^{1}\right)_{\left|\Omega^{n, k_{i}(m)}\right|_{H_{00}}^{\frac{1}{2}\left(\gamma_{i j}^{m}\right)}}\right) \\
& \leq c \mu_{\delta} \sum_{m=1}^{M^{n}} \sum_{i=1}^{p(m)} \sum_{j=1}^{q(i)}\left(\left\|u^{1}-v_{\delta}^{1}\right\|_{H^{1}\left(\tilde{\Delta}_{i j}^{k(m)}\right)}^{2}+\left\|u^{1}-v_{\delta}^{1}\right\|_{H^{1}\left(\Delta_{j}^{k_{i}(m)}\right)}^{2}\right),
\end{aligned}
$$

where $\tilde{\Delta}_{i j}^{k(m)}$ is a triangle contained in $\Delta^{k(m)}$ such that $\gamma_{i j}^{m}$ is an edge of it. By noting that two different $\tilde{\Delta}_{i j}^{k(m)}$ do not intersect for all $i$ and $j$, we derive

$$
\left\|v_{\delta}^{2}\right\|_{H_{\delta}^{1}(\Omega)} \leq c \mu_{\delta}^{\frac{1}{2}}\left(\left\|u-v_{\delta}^{1}\right\|_{H_{\delta}^{1}(\Omega)}+\left\|u-u^{1}\right\|_{H_{\delta}^{1}(\Omega)}\right) .
$$

Moreover, the quantity $\left\|u-u^{1}\right\|_{H_{\delta}^{1}(\Omega)}$ can be bounded by exactly the same arguments as in the first part of the proof, which gives

$$
\left\|v_{\delta}^{2}\right\|_{H_{\delta}^{1}(\Omega)} \leq c \mu_{\delta}^{\frac{1}{2}}\left(\sum_{k=0}^{K^{n}}\left(h^{n, k}\right)^{2\left(s_{k}-1\right)}\|u\|_{H^{s_{k}\left(\Omega^{n, k}\right)}}^{2}\right)^{\frac{1}{2}} .
$$

Taking $v_{\delta}=v_{\delta}^{1}+v_{\delta}^{2}$, noting that it belongs to $\mathbb{X}_{\delta}$ and using (2.17) and (2.18), yields the desired estimate.

Inserting (2.13) and (2.16) into (2.12) leads to the a priori error estimate.

Theorem 2.4. Let us assume the solution u of problem (1.1) such that, for all $n$, each $u_{\mid \Omega^{n, k}}, 0 \leq k \leq K^{n}$, belongs to $H^{s_{k}}\left(\Omega^{n, k}\right), 1<s_{k} \leq \ell+1$. If Assumption A.1 is satisfied, there exists a constant $c$ independent of $\delta$ such that the following error estimate holds between this solution $u$ and the solution $u_{\delta}$ of problem (2.7):

$$
\left\|u-u_{\delta}\right\|_{H_{\delta}^{1}(\Omega)} \leq c \mu_{\delta}^{\frac{1}{2}}\left(\sum_{k=0}^{K^{n}}\left(h^{n, k}\right)^{2\left(s_{k}-1\right)}\|u\|_{H^{s_{k}\left(\Omega^{n, k}\right)}}^{2}\right)^{\frac{1}{2}} .
$$

Remark. Note that, in practice, $\mu_{\delta}$ is most often bounded independently of $\delta$. Moreover, since it only involves local ratios, this independency can easily be enforced in the refinement process.

Since $\Omega$ is a polygon, a minimal value of the regularity exponent $s_{k}$ is known as a function of the regularity of the data when $\Omega^{n, k}$ contains no vertex of $\Omega$, as a function of the regularity of the data and the maximal angles of the vertices contained in $\partial \Omega^{n, k} \cap \partial \Omega$ if not. So, estimate (2.19) allows for a first, a priori, adaptivity of the mesh. But this is not sufficient in most practical situations. So, we must perform the a posteriori analysis of the problem in order to exhibit error indicators which allow for better adaptivity.

\section{THE ERROR INDICATORS AND THE A POSTERIORI ANALYSIS}

In a first step, we introduce some notation in order to define the two kinds of error indicators, linked respectively to the finite elements and to the edges of the skeleton. In a second step, we prove the a posteriori error estimates, first by exhibiting an upper bound for the global error $\left\|u-u_{\delta}\right\|_{H_{\delta}^{1}(\Omega)}$ as a function of the 
indicators, second by estimating each indicator as a function of the local error. We conclude with some remarks.

3.a. The error indicators. Let $\ell^{*}$ stand for a fixed nonnegative integer. For each value of the parameter $\delta$, we introduce the space

$$
\mathbb{Z}_{\delta}=\left\{g_{\delta} \in L^{2}(\Omega) ; \forall K \in \mathcal{T}_{h}^{n}, g_{\delta \mid K} \in \mathcal{P}_{\ell^{*}}(K)\right\} .
$$

And we fix an approximation $f_{\delta}$ of the function $f$ in $\mathbb{Z}_{\delta}$.

Error indicators linked to the finite elements. For each $K$ in $\mathcal{T}_{h}^{n}$, we denote by $\mathcal{E}_{K}$ the set of edges of $K$ which are not contained in $\partial \Omega$. In what follows, $h_{K}$ stands for the diameter of $K$ and $h_{e}$ for the length of any $e$ in $\mathcal{E}_{K}$.

The residual error indicator $\eta_{K}$ associated with any triangle $K$ in $\mathcal{T}_{h}^{n}$ is now defined in a completely standard way (see [20, (1.18)]:

$$
\eta_{K}=h_{K}\left\|f_{\delta}+\Delta u_{\delta}\right\|_{L^{2}(K)}+\frac{1}{2} \sum_{e \in \mathcal{E}_{K}} h_{e}^{\frac{1}{2}}\left\|\left[\partial_{n} u_{\delta}\right]\right\|_{L^{2}(e)},
$$

where, as in Section 2, $\partial_{n}$ denotes the normal derivative on $e$ and [ $\left.\cdot\right]$ the jump through $e$. Note that the term "residual" here means that, when suppressing all the $\delta$ in the previous line, the quantity in the right-hand side is zero.

Error indicators linked to the edges of the skeleton. As previously, we denote by $\mathcal{E}^{m}$ either the edge $\gamma^{m}$ when $\widetilde{W}^{m}$ is chosen equal to $\widetilde{W}_{\mathrm{C}}^{m}$ or the set of the open connected components of the $\gamma^{m} \cap \partial \Omega^{n, k_{i}(m)}, 1 \leq i \leq p(m)$, when $\widetilde{W}^{m}$ is chosen equal to $\widetilde{W}_{\mathrm{F}}^{m}$.

As in [10, (3.3)], for $1 \leq m \leq M^{n}$, we associate with each $e$ in $\mathcal{E}^{m}$ the indicator $\eta_{e}$ defined as

$$
\eta_{e}=\tilde{h}_{e}^{-\frac{1}{2}}\left\|\left[u_{\delta}\right]\right\|_{L^{2}(e)},
$$

where $\tilde{h}_{e}$ denotes the length of $e$ if $\widetilde{W}^{m}$ is equal to $\widetilde{W}_{\mathrm{C}}^{m}$, the largest length of $e^{\prime}=e \cap \partial K$ for $K$ in $\mathcal{T}_{h}^{n, k_{i}(m)}, 1 \leq i \leq p(m)$, if $\widetilde{W}^{m}$ is equal to $\widetilde{W}_{\mathrm{F}}^{m}$. There also, the quantity $\left[u_{\delta}\right]$ vanishes when suppressing the $\delta$.

Remark. It is readily checked that, for all $m, 1 \leq m \leq M^{n}$, such that $p(m)$ is equal to 1 , and for all $e$ in $\mathcal{E}^{m}$, thanks to an inverse inequality [12, Thm. 17.2],

$$
\left\|\left[u_{\delta}\right]\right\|_{H^{\frac{1}{2}(e)}} \leq c \mu_{\delta}^{\frac{1}{2}} \eta_{e},
$$

where $\mu_{\delta}$ is introduced in (2.14). Conversely, thanks to the matching conditions (2.6), we also have

$$
\left(\sum_{e \in \mathcal{E}^{m}} \eta_{e}^{2}\right)^{\frac{1}{2}} \leq c^{\prime}\left\|\left[u_{\delta}\right]\right\|_{H^{\frac{1}{2}}\left(\gamma^{m}\right)} .
$$

However, the quantity $\left\|\left[u_{\delta}\right]\right\|_{H^{\frac{1}{2}}\left(\gamma^{m}\right)}$ is not necessarily defined when $p(m)$ is $>1$, since the jump $\left[u_{\delta}\right]$ has no reason to be continuous on $\gamma_{m}$. Moreover, the norm $\|\cdot\|_{L^{2}(e)}$ is easier to compute that the norm $\|\cdot\|_{H^{\frac{1}{2}(e)}}$.

As a consequence of definitions (3.2) and (3.3), once the discrete solution $u_{\delta}$ is known and the approximation $f_{\delta}$ is fixed, all the error indicators can be computed easily. 
3.b. An upper bound for the error. Since both $H_{0}^{1}(\Omega)$ and $\mathbb{X}_{\delta}$ are contained in the space $\mathbb{X}^{n}$ introduced in Lemma 2.1 , we deduce from the ellipticity property (2.9) that, if Assumption A.1 holds,

$$
\alpha\left\|u-u_{\delta}\right\|_{H_{\delta}^{1}(\Omega)}^{2} \leq a_{\delta}\left(u-u_{\delta}, u-u_{\delta}\right) .
$$

Next, we set $v=u-u_{\delta}$ and we fix an approximation $v_{\delta}$ of $v$ in $\mathbb{X}_{\delta}$. Note that multiplying the first line in (1.1) by $v_{\delta}$ and integrating by parts yields

$$
a_{\delta}\left(u, v_{\delta}\right)=\int_{\Omega} f(\boldsymbol{x}) v_{\delta}(\boldsymbol{x}) d \boldsymbol{x}+\int_{\mathcal{S}^{n}} \partial_{n} u\left[v_{\delta}\right] d \tau .
$$

Subtracting (2.7) and inserting this into the previous line, we obtain

$$
\alpha\left\|u-u_{\delta}\right\|_{H_{\delta}^{1}(\Omega)}^{2} \leq a_{\delta}\left(u-u_{\delta}, v-v_{\delta}\right)+\int_{\mathcal{S}^{n}} \partial_{n} u\left[v_{\delta}\right] d \tau .
$$

Next, we integrate by parts the first term in the right-hand side on each element $K$ of $\mathcal{T}_{h}^{n}$. If $\partial_{n}$ denotes the derivative with respect to the unit outward normal vector of $K$, this leads to

$$
\begin{aligned}
\alpha\left\|u-u_{\delta}\right\|_{H_{\delta}^{1}(\Omega)}^{2} \leq & \sum_{K \in \mathcal{T}_{h}^{n}}\left(\int_{K}\left(f+\Delta u_{\delta}\right)\left(v-v_{\delta}\right) d \boldsymbol{x}+\int_{\partial K} \partial_{n}\left(u-u_{\delta}\right)\left(v-v_{\delta}\right) d \tau\right) \\
& +\int_{\mathcal{S}^{n}} \partial_{n} u\left[v_{\delta}\right] d \tau .
\end{aligned}
$$

Adding and subtracting $f_{\delta}$ and introducing the jump of $\partial_{n}\left(u-u_{\delta}\right)\left(v-v_{\delta}\right)$ on each edge $e$ of $\partial K$ gives

$$
\begin{aligned}
& \alpha\left\|u-u_{\delta}\right\|_{H_{\delta}^{1}(\Omega)}^{2} \\
& \leq \sum_{K \in \mathcal{T}_{h}^{n}}\left(\int_{K}\left(f_{\delta}+\Delta u_{\delta}\right)\left(v-v_{\delta}\right) d \boldsymbol{x}\right. \\
& \left.\quad+\int_{K}\left(f-f_{\delta}\right)\left(v-v_{\delta}\right) d \boldsymbol{x}+\frac{1}{2} \sum_{e \in \mathcal{E}_{K}} \int_{e}\left[\partial_{n}\left(u-u_{\delta}\right)\left(v-v_{\delta}\right)\right] d \tau\right) \\
& \quad+\int_{\mathcal{S}^{n}} \partial_{n} u\left[v_{\delta}\right] d \tau .
\end{aligned}
$$

Consider now an $e$ in $\mathcal{E}_{K}$. If $e$ is not contained in the skeleton $\mathcal{S}^{n}$, it is easy to check that

$$
\int_{e}\left[\partial_{n}\left(u-u_{\delta}\right)\left(v-v_{\delta}\right)\right] d \tau=-\int_{e}\left[\partial_{n} u_{\delta}\right]\left(v-v_{\delta}\right) d \tau
$$

But, when $e$ is contained in $\mathcal{S}^{n}$, a further term appears:

$$
\int_{e}\left[\partial_{n}\left(u-u_{\delta}\right)\left(v-v_{\delta}\right)\right] d \tau=-\int_{e}\left[\partial_{n} u_{\delta}\right]\left(v-v_{\delta}\right) d \tau+\int_{e} \partial_{n}\left(u-u_{\delta}\right)\left[v-v_{\delta}\right] d \tau .
$$


Combining all this leads to

$$
\begin{aligned}
\alpha\left\|u-u_{\delta}\right\|_{H_{\delta}^{1}(\Omega)}^{2} \leq & \sum_{K \in \mathcal{T}_{h}^{n}}\left(\int_{K}\left(f_{\delta}+\Delta u_{\delta}\right)\left(v-v_{\delta}\right) d \boldsymbol{x}\right. \\
& +\int_{K}\left(f-f_{\delta}\right)\left(v-v_{\delta}\right) d \boldsymbol{x} \\
& \left.-\frac{1}{2} \sum_{e \in \mathcal{E}_{K}} \int_{e}\left[\partial_{n} u_{\delta}\right]\left(v-v_{\delta}\right) d \tau\right) \\
& +\sum_{m=1}^{M^{n}} \sum_{e \in \mathcal{E}^{m}}\left(\int_{e} \partial_{n} u\left[v_{\delta}\right] d \tau+\int_{e} \partial_{n}\left(u-u_{\delta}\right)\left[v-v_{\delta}\right] d \tau\right) .
\end{aligned}
$$

In view of this last result, we decide to choose a conforming approximation $v_{\delta}$ of $v$, in the sense that $v_{\delta}$ belongs to $\mathbb{X}_{\delta} \cap H_{0}^{1}(\Omega)$. In this case, the previous estimate writes

$$
\begin{aligned}
\alpha\left\|u-u_{\delta}\right\|_{H_{\delta}^{1}(\Omega)}^{2} \leq & \sum_{K \in \mathcal{T}_{h}^{n}}\left(\int_{K}\left(f_{\delta}+\Delta u_{\delta}\right)\left(v-v_{\delta}\right) d \boldsymbol{x}\right. \\
& +\int_{K}\left(f-f_{\delta}\right)\left(v-v_{\delta}\right) d \boldsymbol{x} \\
& \left.-\frac{1}{2} \sum_{e \in \mathcal{E}_{K}} \int_{e}\left[\partial_{n} u_{\delta}\right]\left(v-v_{\delta}\right) d \tau\right) \\
& -\sum_{m=1}^{M^{n}} \sum_{e \in \mathcal{E}^{m}} \int_{e} \partial_{n}\left(u-u_{\delta}\right)\left[u_{\delta}\right] d \tau
\end{aligned}
$$

Using three times the Cauchy-Schwarz inequality yields

$$
\begin{aligned}
& \alpha\left\|u-u_{\delta}\right\|_{H_{\delta}^{1}(\Omega)} \leq c\left(\sum _ { K \in \mathcal { T } _ { h } ^ { n } } \left(\left\|f_{\delta}+\Delta u_{\delta}\right\|_{L^{2}(K)} \frac{\left\|v-v_{\delta}\right\|_{L^{2}(K)}}{\|v\|_{H_{\delta}^{1}(\Omega)}}\right.\right. \\
&+\left\|f-f_{\delta}\right\|_{L^{2}(K)} \frac{\left\|v-v_{\delta}\right\|_{L^{2}(K)}}{\|v\|_{H_{\delta}^{1}(\Omega)}} \\
&\left.+\frac{1}{2} \sum_{e \in \mathcal{E}_{K}}\left\|\left[\partial_{n} u_{\delta}\right]\right\|_{L^{2}(e)} \frac{\left\|v-v_{\delta}\right\|_{L^{2}(e)}}{\|v\|_{H_{\delta}^{1}(\Omega)}}\right) \\
&\left.+\left|\sum_{m=1}^{M^{n}} \sum_{e \in \mathcal{E}^{m}} \int_{e} \partial_{n}\left(u-u_{\delta}\right)\left[u_{\delta}\right] d \tau\right|^{\frac{1}{2}}\right) .
\end{aligned}
$$

It remains to evaluate the ratios and the last term in the right-hand side.

The first three ratios are linked with the local approximation of nonsmooth functions. So in evaluating them, we rely on the construction of an appropriate operator of Clément's type. We refer to 13] and [4 for previous works on this subject, to [18] and [21] for slightly different operators.

For a while, we consider the coarse approximation space, made of continuous piecewise affine functions

$$
\mathbb{X}_{\delta_{-}}=\left\{v_{\delta} \in H_{0}^{1}(\Omega) ; \forall K \in \mathcal{T}_{h}^{n}, v_{\delta \mid K} \in \mathcal{P}_{1}(K)\right\} .
$$

The proof of the following proposition is very similar to that of Lemma 2.3, but more technical, so we have written it in the Appendix instead. 
Proposition 3.1. There exist an operator $R_{\delta}$ from $\mathbb{X}^{n}$ into $\mathbb{X}_{\delta-}$ and a constant $c$ independent of $\delta$ such that the following estimates hold for all functions $v$ in $\mathbb{X}^{n}$

$$
\sum_{K \in \mathcal{T}_{h}^{n}}\left(h_{K}^{-2}\left\|v-R_{\delta} v\right\|_{L^{2}(K)}^{2}+\sum_{e \in \mathcal{E}_{K}} h_{e}^{-1}\left\|v-R_{\delta} v\right\|_{L^{2}(e)}^{2}\right) \leq c \mu_{\delta}\|v\|_{H_{\delta}^{1}(\Omega)}^{2} .
$$

We now evaluate the last term in the right-hand side of (3.6); however, this requires Assumption A.1 and, in a first step, a further condition that we now state (as already hinted, it does not induce any modification in the previous analysis)

Assumption A.2. The further condition is enforced in the definition of $\mathbb{X}_{\delta}$ : any function $v_{\delta}$ of $\mathbb{X}_{\delta}$ is continuous at the endpoints of all $e$ in $\mathcal{E}^{m}, 1 \leq m \leq M^{n}$.

Proposition 3.2. If Assumptions A.1 and A.2 are satisfied, the following estimate holds for $1 \leq m \leq M^{n}$ and for all $e$ in $\mathcal{E}^{m}$ :

$$
\begin{aligned}
& \left|\int_{e} \partial_{n}\left(u-u_{\delta}\right)\left[u_{\delta}\right] d \tau\right| \leq c \mu_{\delta} \eta_{e} \\
& \left(\left|u-u_{\delta}\right|_{H^{1}\left(K_{e}\right)}+\mu_{\delta}\left(\sum_{K \in \mathcal{T}_{h}^{n}, K \subset K_{e}} h_{K}^{2}\left(\left\|f_{\delta}+\Delta u_{\delta}\right\|_{L^{2}(K)}^{2}+\left\|f-f_{\delta}\right\|_{L^{2}(K)}^{2}\right)\right)^{\frac{1}{2}}\right),
\end{aligned}
$$

where $K_{e}$ is a triangle and $e$ is an edge of $K_{e}$.

Proof. Thanks to Assumption A.2, $\left[u_{\delta}\right]$ vanishes at the endpoints of $e$, hence belongs to $H_{00}^{\frac{1}{2}}(e)$. So, setting $v=u-u_{\delta}$, we have

$$
\int_{e} \partial_{n} v\left[u_{\delta}\right] d \tau \leq\left\|\partial_{n} v\right\|_{H_{00}^{\frac{1}{2}}(e)^{\prime}}\left\|\left[u_{\delta}\right]\right\|_{H_{00}^{\frac{1}{2}}(e)} .
$$

We evaluate separately the two terms in the right-hand side.

1) Going to the reference element and using the Poincaré-Friedrichs inequality on $e$, we have

$$
\left\|\left[u_{\delta}\right]\right\|_{H_{00}^{\frac{1}{2}}(e)} \leq c h_{e}^{\frac{1}{2}}\left\|\left[u_{\delta}\right]\right\|_{H^{1}(e)} .
$$

Since $\left[u_{\delta}\right]$ is polynomial on either one or several intervals of same length $\tilde{h}_{e}$ and the ratio $h_{e} / \tilde{h}_{e}$ is smaller than $\mu_{\delta}$, another inverse inequality leads to

$$
\left\|\left[u_{\delta}\right]\right\|_{H_{00}^{\frac{1}{2}}(e)} \leq c h_{e}^{\frac{1}{2}} \tilde{h}_{e}^{-1}\left\|\left[u_{\delta}\right]\right\|_{L^{2}(e)} \leq c \mu_{\delta} \tilde{h}_{e}^{-\frac{1}{2}}\left\|\left[u_{\delta}\right]\right\|_{L^{2}(e)} .
$$

2) Let $K_{e}$ denote the triangle in $\mathcal{T}_{h}^{n, k(m)}$ such that $e$ is an edge of $K_{e}$ if $\widetilde{W}^{m}=\widetilde{W}_{\mathrm{C}}^{m}$ or the triangle on the other side of $\gamma^{m}$ such that $e$ is an edge of $K_{e}$ if not. In order to estimate

$$
\left\|\partial_{n} v\right\|_{H_{00}^{\frac{1}{2}}(e)^{\prime}}=\sup _{g \in H_{00}^{\frac{1}{2}}(e)} \frac{\left\langle\partial_{n} v, g\right\rangle}{\|g\|_{H_{00}^{\frac{1}{2}}(e)}},
$$

we note that, for any $g$ in $H_{00}^{\frac{1}{2}}(e)$, there exists a lifting $w_{g}$ of $g$ in $H^{1}\left(K_{e}\right)$ which moreover vanishes on $\partial K_{e} \backslash e$ and satisfies (this is proven by going to a reference element)

$$
\left|w_{g}\right|_{H^{1}\left(K_{e}\right)}+h_{K_{e}}^{-1}\left\|w_{g}\right\|_{L^{2}\left(K_{e}\right)} \leq c\|g\|_{H_{00}^{\frac{1}{2}}(e)} .
$$


We have

$$
\left\langle\partial_{n} v, g\right\rangle=\int_{K_{e}} \operatorname{grad} v \cdot \operatorname{grad} w_{g} d \boldsymbol{x}+\int_{K_{e}} \Delta v w_{g} d \boldsymbol{x},
$$

whence

$$
\left\|\partial_{n} v\right\|_{H_{00}^{\frac{1}{2}}(e)^{\prime}} \leq c\left(\left(|v|_{H^{1}\left(K_{e}\right)}+\mu_{\delta}\left(\sum_{K \subset K_{e}} h_{K}^{2}\left(\left\|f_{\delta}+\Delta u_{\delta}\right\|_{L^{2}(K)}^{2}+\left\|f-f_{\delta}\right\|_{L^{2}(K)}^{2}\right)\right)^{\frac{1}{2}}\right) .\right.
$$

Combining all this and using the definition (3.3) of $\eta_{e}$ yield the desired result.

Assumptions A.1 and A.2 are necessary in order to avoid working with quantities that do not belong to $H_{00}^{\frac{1}{2}}(e)$. However, Assumption A.2 is sometimes restrictive for very complex mesh partitions, so we now prove an analogous result when it does not hold. The proof is very similar to that of Proposition 3.2, so we only sketch it.

Corollary 3.3. If Assumption A.1 is satisfied, for any $s, 0<s<\frac{1}{2}$, the following estimate holds for $1 \leq m \leq M^{n}$ and for all e in $\mathcal{E}^{m}$ :

$$
\begin{aligned}
\left|\int_{e} \partial_{n}\left(u-u_{\delta}\right)\left[u_{\delta}\right] d \tau\right| & \\
\leq & \left.c \mu_{\delta} \eta_{e}\left(\tilde{h}_{e}^{s}\left|u-u_{\delta}\right|_{H^{1+s}\left(K_{e}\right)} h_{K}^{2}\left(\left\|f_{\delta}+\Delta u_{\delta}\right\|_{L^{2}(K)}^{2}+\left\|f-f_{\delta}\right\|_{L^{2}(K)}^{2}\right)\right)^{\frac{1}{2}}\right), \\
& \quad+\mu_{\delta}\left(\sum_{K \in \mathcal{T}_{h}^{n}, K \subset K_{e}}\right.
\end{aligned}
$$

where $K_{e}$ is a triangle and $e$ is an edge of $K_{e}$.

Proof. For each $e$ in $\mathcal{E}^{m}$, setting as previously $v=u-u_{\delta}$, we have

$$
\int_{e} \partial_{n} v\left[u_{\delta}\right] d \tau \leq\left\|\partial_{n} v\right\|_{H^{s-\frac{1}{2}(e)}}\left\|\left[u_{\delta}\right]\right\|_{H^{\frac{1}{2}-s}(e)} .
$$

1) Thanks to an inverse inequality, we have

$$
\left\|\left[u_{\delta}\right]\right\|_{H^{\frac{1}{2}-s}(e)} \leq \mu_{\delta} \tilde{h}_{e}^{s-\frac{1}{2}}\left\|\left[u_{\delta}\right]\right\|_{L^{2}(e)} .
$$

2) It remains to evaluate

$$
\left\|\partial_{n} v\right\|_{H^{s-\frac{1}{2}}(e)}=\sup _{g \in H^{\frac{1}{2}-s}(e)} \frac{\left\langle\partial_{n} v, g\right\rangle}{\|g\|_{H^{\frac{1}{2}-s}(e)}} .
$$

For any $g$ in $H^{\frac{1}{2}-s}(e)$, there exists a lifting $w_{g}$ of $g$ in $H^{1-s}\left(K_{e}\right)$ which moreover vanishes on $\partial K_{e} \backslash e$ and satisfies

$$
\left|w_{g}\right|_{H^{1-s}\left(K_{e}\right)}+h_{K_{e}}^{s-1}\left\|w_{g}\right\|_{L^{2}\left(K_{e}\right)} \leq c\|g\|_{H^{\frac{1}{2}-s}(e)} .
$$

We deduce from (3.11) that

$$
\begin{aligned}
& \left\|\partial_{n} v\right\|_{H^{\frac{1}{2}-s}(e)} \leq c|v|_{H^{1+s}\left(K_{e}\right)} \\
& \quad+c^{\prime} \mu_{\delta}\left(\sum_{K \subset K_{e}} h_{K}^{2(1-s)}\left(\left\|f_{\delta}+\Delta u_{\delta}\right\|_{L^{2}(K)}^{2}+\left\|f-f_{\delta}\right\|_{L^{2}(K)}^{2}\right)\right)^{\frac{1}{2}} .
\end{aligned}
$$

This gives the desired result.

Inserting (3.8) and (3.9) (or (3.12)) into (3.6) leads to the a posteriori error estimate. 
Theorem 3.4. If Assumptions A.1 and A.2 hold, there exists a constant c independent of $\delta$ such the following error estimate holds between the solution $u$ of problem (1.1) and the solution $u_{\delta}$ of problem (2.7):

$$
\left\|u-u_{\delta}\right\|_{H_{\delta}^{1}(\Omega)} \leq c \mu_{\delta}\left(\sum_{K \in \mathcal{T}_{h}^{n}}\left(\eta_{K}^{2}+h_{K}^{2}\left\|f-f_{\delta}\right\|_{L^{2}(K)}^{2}\right)+\sum_{m=1}^{M^{n}} \sum_{e \in \mathcal{E}^{m}} \eta_{e}^{2}\right)^{\frac{1}{2}} .
$$

Corollary 3.5. If Assumption A.1 holds, for any $s, 0<s<\frac{1}{2}$, let $\lambda_{\delta s}(u)$ be the smallest constant, depending on the solution $u$ of problem (1.1) and the solution $u_{\delta}$ of problem (2.7), such that

$$
\left(\sum_{K \in \mathcal{T}_{h}^{n}} h_{K}^{2 s}\left|u-u_{\delta}\right|_{H^{1+s}(K)}^{2}\right)^{\frac{1}{2}} \leq \lambda_{\delta s}(u)\left\|u-u_{\delta}\right\|_{H_{\delta}^{1}(\Omega)} .
$$

There exists a constant $c$ independent of $\delta$ such the following error estimate holds between these solutions:

$$
\left\|u-u_{\delta}\right\|_{H_{\delta}^{1}(\Omega)} \leq c \mu_{\delta}\left(\sum_{K \in \mathcal{T}_{h}^{n}}\left(\eta_{K}^{2}+h_{K}^{2}\left\|f-f_{\delta}\right\|_{L^{2}(K)}^{2}\right)+\left(1+\lambda_{\delta s}\right) \sum_{m=1}^{M^{n}} \sum_{e \in \mathcal{E}^{m}} \eta_{e}^{2}\right)^{\frac{1}{2}} .
$$

Assume that the $\mu_{\delta}$ are bounded independently of $\delta$, which is most often the case in practical situations. Then, when Assumption A.2 holds, we obtain estimate (3.13), which is fully optimal. When it does not hold, we only derive estimate (3.15) which is not optimal. Note however that the constant $\lambda_{\delta s}(u)$ tends to 1 when $s$ tends to 0 .

3.c. An upper bound for the indicators. The idea is now to bound both the $\eta_{K}$ and the $\eta_{e}$ as a function of the norm of the error $u-u_{\delta}$, both locally and globally.

In a first step, we fix a function $w$ in $H_{0}^{1}(\Omega)$ and we compute by integration by parts

$$
\begin{aligned}
& \sum_{K \in \mathcal{T}_{h}^{n}} \int_{K} \operatorname{grad}\left(u-u_{\delta}\right) \cdot \operatorname{grad} w d \boldsymbol{x} \\
& \quad=\sum_{K \in \mathcal{T}_{h}^{n}}\left(\int_{K}\left(f+\Delta u_{\delta}\right) w d \boldsymbol{x}+\int_{\partial K} \partial_{n}\left(u-u_{\delta}\right) w d \tau\right),
\end{aligned}
$$

whence

$$
\begin{aligned}
& \sum_{K \in \mathcal{T}_{h}^{n}} \int_{K} \operatorname{grad}\left(u-u_{\delta}\right) \cdot \operatorname{grad} w d \boldsymbol{x} \\
& \quad=\sum_{K \in \mathcal{T}_{h}^{n}}\left(\int_{K}\left(f_{\delta}+\Delta u_{\delta}\right) w d \boldsymbol{x}+\int_{K}\left(f-f_{\delta}\right) w d \boldsymbol{x}-\frac{1}{2} \sum_{e \in \mathcal{E}_{K}} \int_{e}\left[\partial_{n} u_{\delta}\right] w d \tau\right)
\end{aligned}
$$

Indeed, as is standard for residual type indicators, proving the first estimates relies on appropriate choices of the function $w$ in (3.16).

With each triangle $K$ in $\mathcal{T}_{h}^{n}$, we associate the bubble function $\psi_{K}$ equal to the product of the three barycentric coordinates on $K$. For each edge $e$ in $\mathcal{E}_{K}$, we also introduce the bubble function $\psi_{e}$ related to $e$, i.e., equal to the product of the two barycentric coordinates corresponding to the endpoints of $e$. Finally, on a reference 

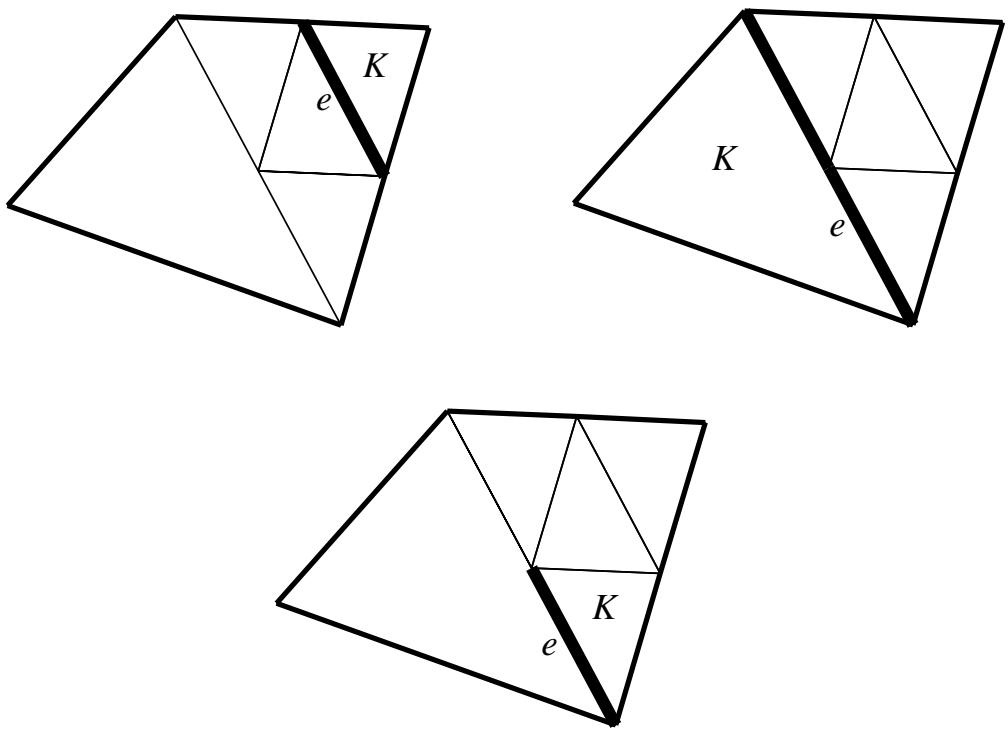

FIGURE 4.

triangle $\hat{K}$, we fix a lifting operator $\hat{P}$ from polynomial traces on an edge $\hat{e}$ of $\hat{K}$ that vanish at the endpoints of $\hat{e}$ into polynomials on $\hat{K}$ that vanish on $\partial \hat{K} \backslash \hat{e}$. A similar operator $P_{K, e}$ is then built from $\hat{P}$ by affine transformation for all triangles $K$ and all edges $e$ of $K$.

Proposition 3.6. There exists a constant $c$ independent of $\delta$ such that the following estimate holds for all $K$ in $\mathcal{T}_{h}^{n}$ :

$$
\eta_{K} \leq c\left(\left\|u-u_{\delta}\right\|_{H_{\delta}^{1}\left(\Xi_{K}\right)}+\mu_{\delta}\left(\sum_{K^{\prime} \subset \Xi_{K}} h_{K^{\prime}}^{2}\left\|f-f_{\delta}\right\|_{L^{2}\left(K^{\prime}\right)}^{2}\right)^{\frac{1}{2}}\right),
$$

where $\Xi_{K}$ is the union of at most four triangles that contain an edge of $K$.

Proof. Bounding the first term in $\eta_{K}$ is fully standard (see [20 §1.2]): taking $w$ in (3.16) equal to

$$
w= \begin{cases}\left(f_{\delta}+\Delta u_{\delta}\right) \psi_{K} & \text { on } K, \\ 0 & \text { elsewhere }\end{cases}
$$

and using standard inverse inequalities lead to the estimate

$$
h_{K}\left\|f_{\delta}+\Delta u_{\delta}\right\|_{L^{2}(K)} \leq c\left(\left|u-u_{\delta}\right|_{H^{1}(K)}+h_{K}\left\|f-f_{\delta}\right\|_{L^{2}(K)}\right) .
$$

Now let $e$ be an edge in $\mathcal{E}_{K}$. Three cases occur, as illustrated in Figure 4.

1) If $e$ is not contained in $\overline{\mathcal{S}}^{n}$, it is the edge of two triangles $K$ and $K^{\prime}$ of the same $\mathcal{T}_{h}^{n, k}$. We then take $w$ in (3.16) equal to

$$
w= \begin{cases}P_{K, e}\left(\left[\partial_{n} u_{\delta}\right] \psi_{e}\right) & \text { on } K, \\ P_{K^{\prime}, e}\left(\left[\partial_{n} u_{\delta}\right] \psi_{e}\right) & \text { on } K^{\prime}, \\ 0 & \text { elsewhere. }\end{cases}
$$


This gives

$$
\begin{aligned}
& \left\|\left[\partial_{n} u_{\delta}\right] \psi_{e}^{\frac{1}{2}}\right\|_{L^{2}(e)}^{2} \\
& \leq\left.\sum_{\kappa \in\left\{K, K^{\prime}\right\}}\left(\left|u-u_{\delta}\right|_{H^{1}(\kappa)} \mid P_{\kappa, e}\left(\left[\partial_{n} u_{\delta}\right] \psi_{e}\right]\right)\right|_{H^{1}(\kappa)} \\
& \left.\quad+\left(\left\|f_{\delta}+\Delta u_{\delta}\right\|_{L^{2}(\kappa)}+\left\|f-f_{\delta}\right\|_{L^{2}(\kappa)}\right) \| P_{\kappa, e}\left(\left[\partial_{n} u_{\delta}\right] \psi_{e}\right]\right) \|_{L^{2}(\kappa)} .
\end{aligned}
$$

There also, we prove the following inequalities by going to a reference element

$$
\begin{gathered}
\left\|\left[\partial_{n} u_{\delta}\right]\right\|_{L^{2}(e)} \leq c\left\|\left[\partial_{n} u_{\delta}\right] \psi_{e}^{\frac{1}{2}}\right\|_{L^{2}(e)}, \\
\left.\left.\mid P_{\kappa, e}\left(\left[\partial_{n} u_{\delta}\right] \psi_{e}\right]\right)\left.\right|_{H^{1}(\kappa)}+h_{e}^{-1} \| P_{\kappa, e}\left(\left[\partial_{n} u_{\delta}\right] \psi_{e}\right]\right)\left\|_{L^{2}(\kappa)} \leq c h_{e}^{-\frac{1}{2}}\right\|\left[\partial_{n} u_{\delta}\right] \|_{L^{2}(e)} .
\end{gathered}
$$

By noting that $c h_{\kappa} \leq h_{e} \leq h_{\kappa}$, we obtain

$$
\begin{aligned}
& h_{e}^{\frac{1}{2}}\left\|\left[\partial_{n} u_{\delta}\right]\right\|_{L^{2}(e)} \\
& \quad \leq c \sum_{\kappa \in\left\{K, K^{\prime}\right\}}\left(\left|u-u_{\delta}\right|_{H^{1}(\kappa)}+h_{\kappa}\left\|f-f_{\delta}\right\|_{L^{2}(\kappa)}+h_{\kappa}\left\|f_{\delta}+\Delta u_{\delta}\right\|_{L^{2}(\kappa)}\right) .
\end{aligned}
$$

2) If $e$ is contained in a $\bar{\gamma}^{m}$ and $K$ is contained in $\mathcal{T}_{h}^{n, k(m)}$, we denote by $K^{\prime}$ the triangle on the other "side" of $\gamma^{m}$, i.e., the triangle obtained by cutting an element of $\mathcal{T}_{h}^{0}$ into $2^{2 k(m)}$ subtriangles. The same proof as above leads to (3.19); however, a further $\mu_{\delta}$ appears when comparing $h_{K^{\prime}}$ with the diameters of the triangles of the $\mathcal{T}_{h}^{n, k_{i}(m)}$ contained in it.

3) If $e$ is contained in a $\bar{\gamma}^{m}$ and $K$ is contained in a $\mathcal{T}_{h}^{n, k_{i}(m)}$, we denote by $K^{\prime}$ the triangle in $\mathcal{T}_{h}^{k(m)}$ such that $e$ is contained in an edge $e^{\prime}$ of $K^{\prime}$. We extend without change of notation $\left[\partial_{n} u_{\delta}\right] \psi_{e}$ by zero to $e^{\prime}$ and we make the same choice as previously, which gives (3.19).

As a conclusion, combining (3.18) and (3.19) gives the desired bound for $\eta_{K}$.

Taking the sum of the square of estimate (3.17) and summing up on the $K$ that have no edges on $\mathcal{S}^{n}$ leads to a global estimate (indeed, parts of the $\Xi_{K}$ appear a finite number of times in the sum), and this is also true for the $K$ that have an edge in $\bar{\gamma}^{m}$ but belong to $\mathcal{T}_{h}^{n, k(m)}$. For the remaining triangles, we must make a more global choice of $w$, with support in a neighbourhood of $\gamma^{m}$, in order to bound the quantity

$$
\sum_{e \subset \bar{\gamma}^{m}} h_{e}\left\|\left[\partial_{n} u_{\delta}\right]\right\|_{L^{2}(e)}^{2} .
$$

This leads to the following corollary.

Corollary 3.7. There exists a constant $c$ independent of $\delta$ such that the following estimate holds:

$$
\sum_{K \in \mathcal{T}_{h}^{n}} \eta_{K}^{2} \leq c\left(\left\|u-u_{\delta}\right\|_{H_{\delta}^{1}(\Omega)}^{2}+\mu_{\delta}^{2} \sum_{K \in \mathcal{T}_{h}^{n}} h_{K}^{2}\left\|f-f_{\delta}\right\|_{L^{2}(K)}^{2}\right) .
$$

It remains to bound the $\eta_{e}$. The argument here is much simpler. 
Proposition 3.8. If Assumption A.1 is satisfied, there exists a constant $c$ independent of $\delta$ such that the following estimate holds for all $m, 1 \leq m \leq M^{n}$, and for all e in $\mathcal{E}^{m}$ :

$$
\eta_{e} \leq c\left\|u-u_{\delta}\right\|_{H_{\delta}^{1}\left(\Xi_{e}\right)},
$$

where $\Xi_{e}$ is the union of the triangle $K$ in $\Omega^{n, k(m)}$ and a triangle $K^{\prime}$ on the other side of $\gamma^{m}$ such that $e$ is an edge of $K$ and $K^{\prime}$.

Proof. We first consider the case where $\widetilde{W}^{m}$ is taken equal to $\widetilde{W}_{\mathrm{F}}^{m}$, so that $e$ coincides with a connected component of the intersections $\bar{\gamma}^{m} \cap \partial \Omega^{n, k_{i}(m)}$. Denoting by $\bar{u}_{e}$ the projection of $\left[u_{\delta}\right]$ onto $\widetilde{W}^{m}(e)$, thanks to the matching condition (2.6) we have

$$
\left\|\left[u_{\delta}\right]\right\|_{L^{2}(e)}^{2}=\int_{e}\left(\left[u_{\delta}\right]-\bar{u}_{e}\right)^{2} d \tau \leq c \tilde{h}_{e}\left|\left[u_{\delta}\right]\right|_{H^{\frac{1}{2}}(e)}^{2} .
$$

This yields

$$
\tilde{h}_{e}^{-\frac{1}{2}}\left\|\left[u_{\delta}\right]\right\|_{L^{2}(e)} \leq c\left|\left[u-u_{\delta}\right]\right|_{H^{\frac{1}{2}}(e)} .
$$

It is readily checked by going to a reference triangle that the trace operator is continuous from $H^{1}(K)$ into $H^{\frac{1}{2}}(e)$, with norm bounded independently of $K$, where $e$ is a part of its boundary. So, we derive, with obvious notation for $K$ and $K^{\prime}$,

$$
\tilde{h}_{e}^{-\frac{1}{2}}\left\|\left[u_{\delta}\right]\right\|_{L^{2}(e)} \leq c\left(\left|u-u_{\delta}\right|_{H^{1}(K)}+\left|u-u_{\delta}\right|_{H^{1}\left(K^{\prime}\right)}\right) .
$$

When $\widetilde{W}^{m}$ is taken equal to $\widetilde{W}_{\mathrm{C}}^{m}, p(m)$ is equal to 1 by assumption, so that the same arguments as above still work.

There, the global estimate is directly derived from the local ones.

Corollary 3.9. If Assumption A.1 is satisfied, there exists a constant $c$ independent of $\delta$ such that the following estimate holds:

$$
\sum_{m=1}^{M^{n}} \sum_{e \in \mathcal{E}^{m}} \eta_{e}^{2} \leq c\left\|u-u_{\delta}\right\|_{H_{\delta}^{1}(\Omega)}^{2} .
$$

3.d. Concluding remarks. When combining estimates (3.20) and (3.22) and comparing with (3.13), if $\mu_{\delta}$ are bounded independently of $\delta$ (which is most often the case and can be enforced in the algorithm if not), we observe that, up to the terms $h_{K}\left\|f-f_{\delta}\right\|_{L^{2}(K)}$, the error $\left\|u-u_{\delta}\right\|_{H_{\delta}^{1}(\Omega)}$ is equivalent to the Hilbertian sum of the indicators. When the data are locally smooth, the terms $h_{K}\left\|f-f_{\delta}\right\|_{L^{2}(K)}$ can be neglected for an appropriate value of $\ell^{*}$, so that this sum provides an optimal global representation of the error. Moreover, from the local estimates (3.17) and (3.21), it can be hoped that the family of error indicators provides a correct local representation of it, hence is an appropriate tool for adaptivity. This is checked in the following numerical experiments.

\section{Some numerical EXPERIMENTS}

We first explain how problem (2.7) can be solved efficiently. Next we describe an adaptivity algorithm relying on the indicators $\eta_{K}$ and $\eta_{e}$. We conclude with some numerical tests. 
4.a. A way for solving the discrete problem. The key idea for solving the mortar discrete problem (2.7) is due to [2]: it consists in enforcing the matching conditions (2.6) by introducing a Lagrange multiplier. More precisely, let $\mathbb{Y}_{\delta}$ stand for the space of functions $v_{\delta}$ such that

- their restrictions to each $\Omega^{n, k}, 0 \leq k \leq K^{n}$, belong to $X^{n, k}$,

- they vanish on $\partial \Omega$.

Next, for simplicity, we assume that the $\gamma^{m}$ for which $\widetilde{W}^{m}$ is equal to $\widetilde{W}_{\mathrm{C}}^{m}$ are numbered from 1 to $M_{*}^{n}$ and we set:

$$
\mathbb{M}_{\delta}=\left(\prod_{m=1}^{M_{*}^{n}} \widetilde{W}_{\mathrm{C}}^{m}\right) \times\left(\prod_{m=M_{*}^{n}+1}^{M^{n}} \prod_{e \in \mathcal{E}^{m}} \widetilde{W}^{m}(e)\right) .
$$

We now consider the problem:

Find $\left(u_{\delta}, \lambda_{\delta}\right)$ in $\mathbb{Y}_{\delta} \times \mathbb{M}_{\delta}$ such that

$$
\begin{array}{ll}
\forall v_{\delta} \in \mathbb{Y}_{\delta}, & a_{\delta}\left(u_{\delta}, v_{\delta}\right)+b_{\delta}\left(v_{\delta}, \lambda_{\delta}\right)=\int_{\Omega} f(\boldsymbol{x}) v_{\delta}(\boldsymbol{x}) d \boldsymbol{x}, \\
\forall \mu_{\delta} \in \mathbb{M}_{\delta}, & b_{\delta}\left(u_{\delta}, \mu_{\delta}\right)=0
\end{array}
$$

where the bilinear form $b_{\delta}(\cdot, \cdot)$ is defined by

$$
\begin{aligned}
b_{\delta}\left(v_{\delta}, \mu_{\delta}\right)=\sum_{m=1}^{M_{*}^{n}} \int_{\gamma^{m}}\left[v_{\delta}\right](\tau) \mu_{m}(\tau) d \tau+\sum_{m=M_{*}^{n}+1}^{M^{n}} \sum_{e \in \mathcal{E}^{m}} \int_{e}\left[v_{\delta}\right](\tau) \mu_{m, e}(\tau) d \tau, \\
\text { with } \quad \mu_{\delta}=\left(\mu_{m}, \mu_{m, e}\right) .
\end{aligned}
$$

Then it can be checked [2] that if $u_{\delta}$ denotes the solution of problem (2.7), then there exists a unique $\lambda_{\delta}$ such that the pair $\left(u_{\delta}, \lambda_{\delta}\right)$ is the solution of (4.2).

Remark. The definition $(4.3)$ of $b_{\delta}(\cdot, \cdot)$ seems rather complicated. However, if Assumption A.1 holds, the jump $\left[v_{\delta}\right]$ in each integral appearing in this definition only involves the values of $v_{\delta}$ on two subdomains $\Omega^{n, k}$.

Problem (4.2) is of saddle-point type. Hence, with obvious notation, it is equivalent to a square linear system of the type

$$
\left(\begin{array}{cc}
A & B \\
B^{T} & 0
\end{array}\right)\left(\begin{array}{l}
U \\
\Lambda
\end{array}\right)=\left(\begin{array}{c}
F \\
0
\end{array}\right)
$$

The matrix $A$ is square and symmetric positive definite. It is block diagonal, made of $K^{n}+1$ blocks. The matrix $B$ is much smaller since it only involves the traces on one-dimensional edges, and $B^{T}$ stands for its transposed matrix. So the global matrix is symmetric.

To solve problem (4.2) or equivalently (4.4), for simplicity and since we only treat academic problems, we use a direct Gauss factorization of Crout type of the global matrix (see [16] Prop. 5.10.1]). However, for more realistic situations, system (4.4) can be solved via Uzawa's algorithm combined with the preconditioned conjugate gradient method. 
4.b. The adaptivity algorithm. On the "coarse" conforming triangulation $\mathcal{T}_{h}^{0}$, we solve system (4.4) in the simpler case $B=0$. Next we compute the $\eta_{K}, K \in \mathcal{T}_{h}^{0}$, their mean value $\bar{\eta}^{0}$, and we perform Step 1 of adaptivity (with $n=0$ ). Let $\rho$ be a fixed constant.

Step 1. For all $K$ in $\mathcal{T}_{h}^{n}$, there exists an integer $k$ such that

$$
2^{k} \rho \bar{\eta}^{n} \leq \eta_{K}<2^{k+1} \rho \bar{\eta}^{n} .
$$

If $k$ is nonnegative, we cut the triangle $K$ into $2^{2(k+1)}$ equal subtriangles by iteratively joining the middle of the edges. This allows for defining an intermediary skeleton $\mathcal{S}_{*}^{n}$.

This leads to a nonconforming triangulation $\mathcal{T}_{h}^{1}$ with skeleton $\mathcal{S}^{1}=\mathcal{S}_{*}^{0}$. Next, assuming that the triangulation $\mathcal{T}_{h}^{n}$ is known, we solve the corresponding system (4.4). We compute the $\eta_{K}, K \in \mathcal{T}_{h}^{n}$, and their mean value $\bar{\eta}^{n}$, next the $\eta_{e}, e \in \mathcal{E}^{m}$, $1 \leq m \leq M^{n}$, and their mean value $\bar{\eta}_{*}^{n}$. Finally, we perform Steps 1 and 2 of adaptivity.

Step 2. We only consider the $e$ in $\mathcal{E}^{m}, 1 \leq m \leq M^{n}$, such that

$$
\eta^{e} \geq 2 \max \left\{\bar{\eta}^{n}, \bar{\eta}_{*}^{n}\right\} .
$$

If this edge remains in $\mathcal{S}_{*}^{n}$ after Step 1, we cut the triangles on both sides of $e$, such that $e$ becomes "conforming", i.e., it is no longer contained in the next skeleton $\mathcal{S}^{n+1}$

However, in our numercial tests, we choose to take $\widetilde{W}^{m}$ equal to $\widetilde{W}_{\mathrm{F}}^{m}$, so that the $\eta_{e}$ are most often neglectable with respect to the $\eta_{K}$ and inequality (4.6) is never satisfied. We refer to [11] for a recent experiment in a different context, where the two choices of $\widetilde{W}^{m}$ are compared and lead to nearly the same global error.

We take $\rho$ equal to 1.1. We stop the algorithm either after a finite number of iterations (for instance 2 or 3 ) or when the following condition is satisfied

$$
\sum_{K \in \mathcal{T}_{h}^{n}} \eta_{K}^{2}+\sum_{m=1}^{M^{n}} \sum_{e \in \mathcal{E}^{m}} \eta_{e}^{2} \leq \varepsilon^{2},
$$

for a given tolerance $\varepsilon$.

Remark. In view of (4.5), Step 1 of our algorithm can be improved as follows, when keeping in memory a finite number of previous triangulations $\mathcal{T}_{h}^{n-1}, \ldots, \mathcal{T}_{h}^{n-q}$ : when $k$ is negative for all $K$ contained in a larger triangle $K^{\prime}$ of one of these triangulations, these triangles are replaced by $K^{\prime}$ in the new triangulation.

4.c. Numerical experiments. The following tests are implemented on a mortar extension of the finite element code FreeFem + , written in $\mathrm{C}++$ language (see [15]). Piecewise quadratic functions are used in all the tests $(\ell=2)$.

We first work with the $L$-shaped domain $\Omega=] 0,1\left[2 \backslash\left[\frac{1}{4}, 1\left[^{2}\right.\right.\right.$, with data $f=1$. Figure 5 represents the successive meshes $\mathcal{T}_{h}^{n}$, for $n=0, \ldots, 5$. Note that the dimension of $\mathbb{X}_{\delta}$ increases from 89 for $n=0$ to 1671 for $n=5$.

Figure 6 represents the curves of isovalues of the error indicators associated with the elements $K$ of the six meshes $\mathcal{T}_{h}^{n}, n=0, \ldots, 5$.

In Table 1, we present, for $n=0, \ldots, 5$, the number $N_{T}^{n}$ of triangles in $\mathcal{T}_{h}^{n}$, the number $M^{n}$ of mortars, the dimension $\operatorname{dim} \mathbb{X}_{\delta}$ of the corresponding space $\mathbb{X}_{\delta}$ and 

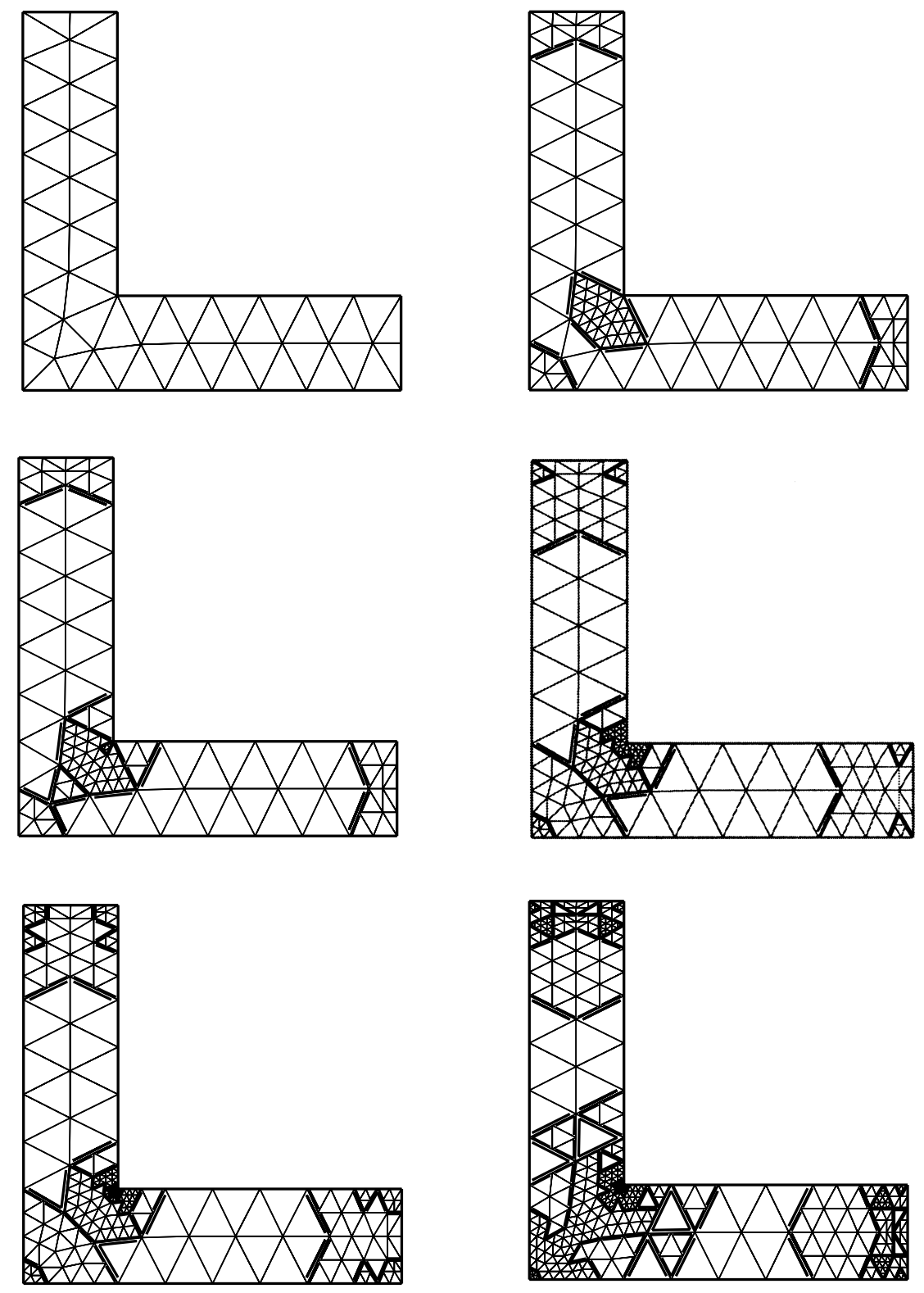

FiguRE 5 .

the quantity

$$
\eta_{\text {norm }}^{n}=\left(\sum_{K \in \mathcal{T}_{h}^{n}} \eta_{K}^{2}+\sum_{m=1}^{M^{n}} \sum_{e \in \mathcal{E}^{m}} \eta_{e}^{2}\right)^{\frac{1}{2}} .
$$

Figure 7 presents the curves of isovalues of the discrete solution, computed on the mesh $\mathcal{T}_{h}^{5}$. 

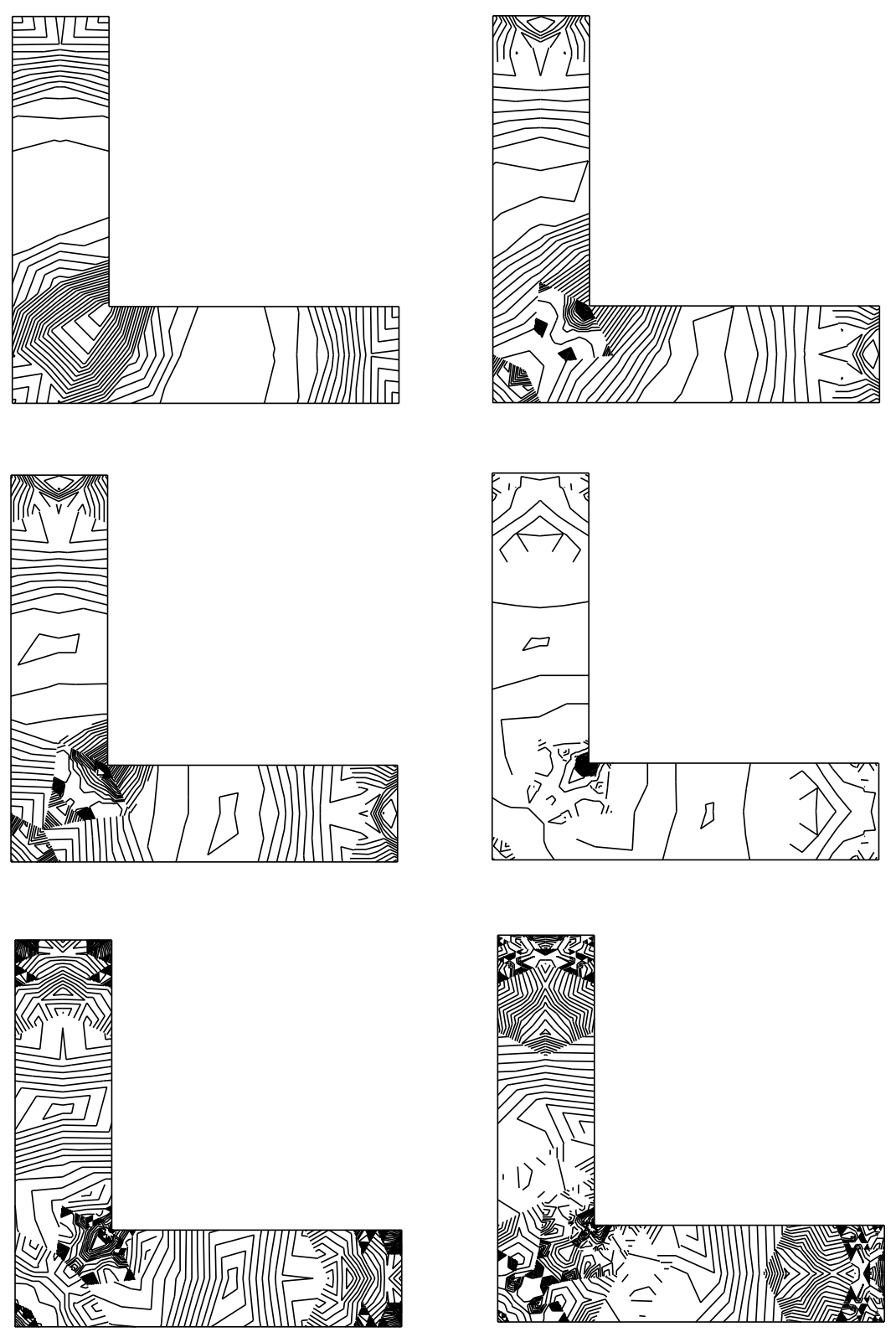

FiguRE 6.

In a second step, we work with the square $\Omega=]-1,1[2$. The function $f$ here is of Heaviside's type:

$$
f(x, y)= \begin{cases}100 & \text { if }(x, y) \in] 0, \frac{1}{2}\left[^{2}\right. \\ 0 & \text { elsewhere }\end{cases}
$$

Figure 8 represents the successive meshes $\mathcal{T}_{h}^{n}$, for $n=0, \ldots, 5$. 


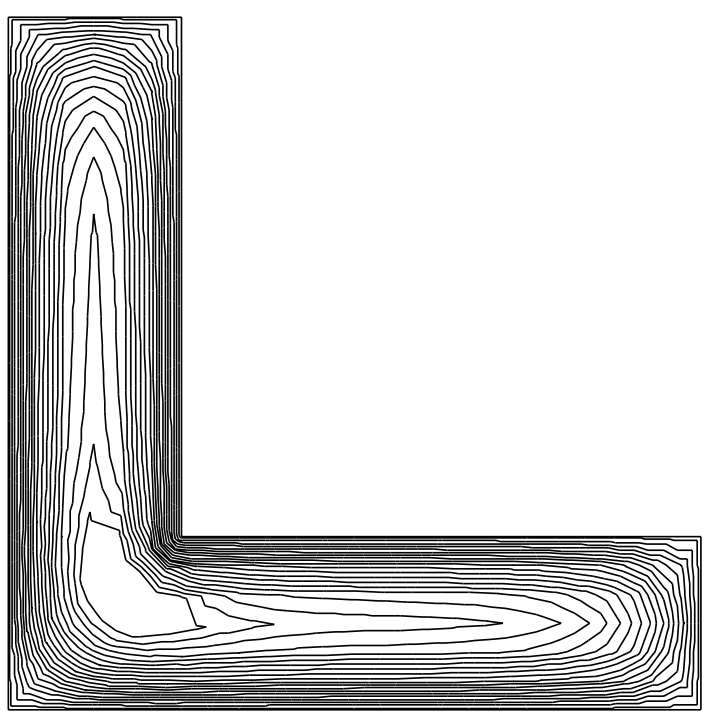

FiguRe 7.

TABLE 1.

\begin{tabular}{|c|c|c|c|c|c|c|}
\hline$n$ & 0 & 1 & 2 & 3 & 4 & 5 \\
\hline$N_{T}^{n}$ & 60 & 135 & 147 & 243 & 495 & 741 \\
$M^{n}$ & 0 & 11 & 21 & 33 & 63 & 129 \\
$\operatorname{dim} \mathbb{X}_{\delta}$ & 89 & 261 & 297 & 499 & 1065 & 1671 \\
$\eta_{\text {norm }}^{n}$ & 0.0323 & 0.0114 & 0.0102 & 0.0073 & 0.0042 & 0.0023 \\
\hline
\end{tabular}

TABLE 2 .

\begin{tabular}{|c|c|c|c|c|c|c|}
\hline$n$ & 0 & 1 & 2 & 3 & 4 & 5 \\
\hline$N_{T}^{n}$ & 178 & 481 & 496 & 538 & 652 & 820 \\
$M^{n}$ & 0 & 43 & 45 & 54 & 97 & 126 \\
$\operatorname{dim} \mathbb{X}_{\delta}$ & 321 & 1027 & 1058 & 1155 & 1468 & 1869 \\
$\eta_{\text {norm }}^{n}$ & 5.1412 & 1.1833 & 0.9704 & 0.7629 & 0.5419 & 0.4155 \\
\hline
\end{tabular}

TABLE 3 .

\begin{tabular}{|c|c|c|c|c|c|c|}
\hline$n$ & 0 & 1 & 2 & 3 & 4 & 5 \\
\hline$N_{T}^{n}$ & 178 & 456 & 499 & 663 & 669 & 1158 \\
$\operatorname{dim} \mathbb{X}_{\delta}$ & 321 & 877 & 960 & 1286 & 1298 & 2269 \\
$\eta_{\text {norm }}^{n}$ & 5.1412 & 1.3882 & 1.1368 & 0.6987 & 0.6906 & 0.4256 \\
\hline
\end{tabular}

In order to compare the convergence results with those obtained by conforming discretizations, we give in Figure 9 the conforming meshes $\mathcal{T}_{h}^{n}$, for $n=0, \ldots, 5$, where the adaptivity relies on the same indicators as above and the following technique:

- The initial mesh $\mathcal{T}_{h}^{0}$ is the same as before. 

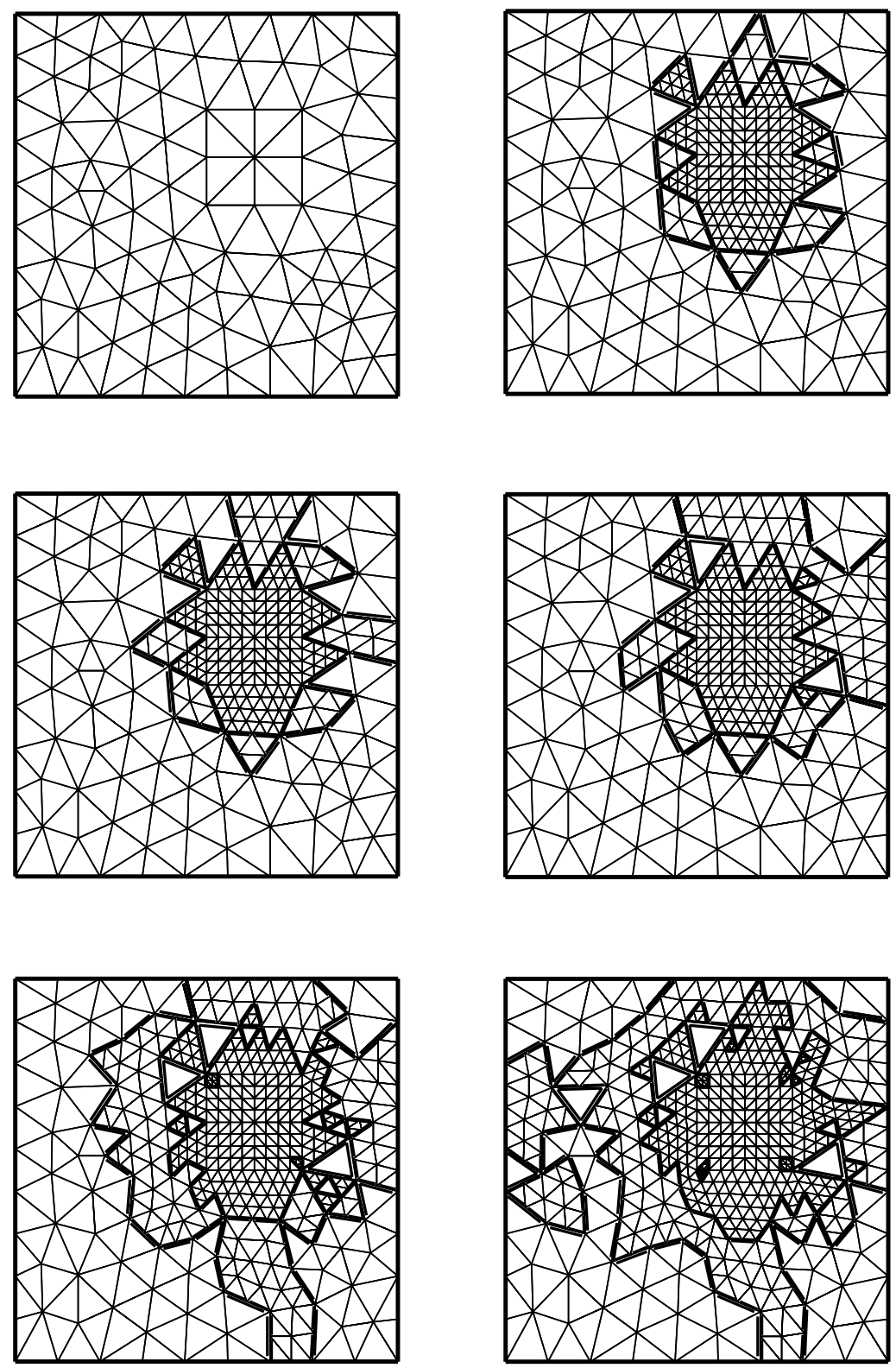

FiguRE 8.

- Once the indicators for $\mathcal{T}_{h}^{n}$ are known, the corners of the new triangles are derived from Steps 1 and 2 of adaptivity and a conforming mesh $\mathcal{T}_{h}{ }^{n+1}$ based on these corners is built by Delaunay's algorithm.

We give values of the same parameters $N_{T}^{n}, M^{n}, \operatorname{dim} \mathbb{X}_{\delta}$ and $\eta_{\text {norm }}^{n}$ as before for the mortar case in Table 2, the values of $N_{T}^{n}$, $\operatorname{dim} \mathbb{X}_{\delta}$ and $\eta_{\text {norm }}^{n}$ for the conforming case in Table 3. 

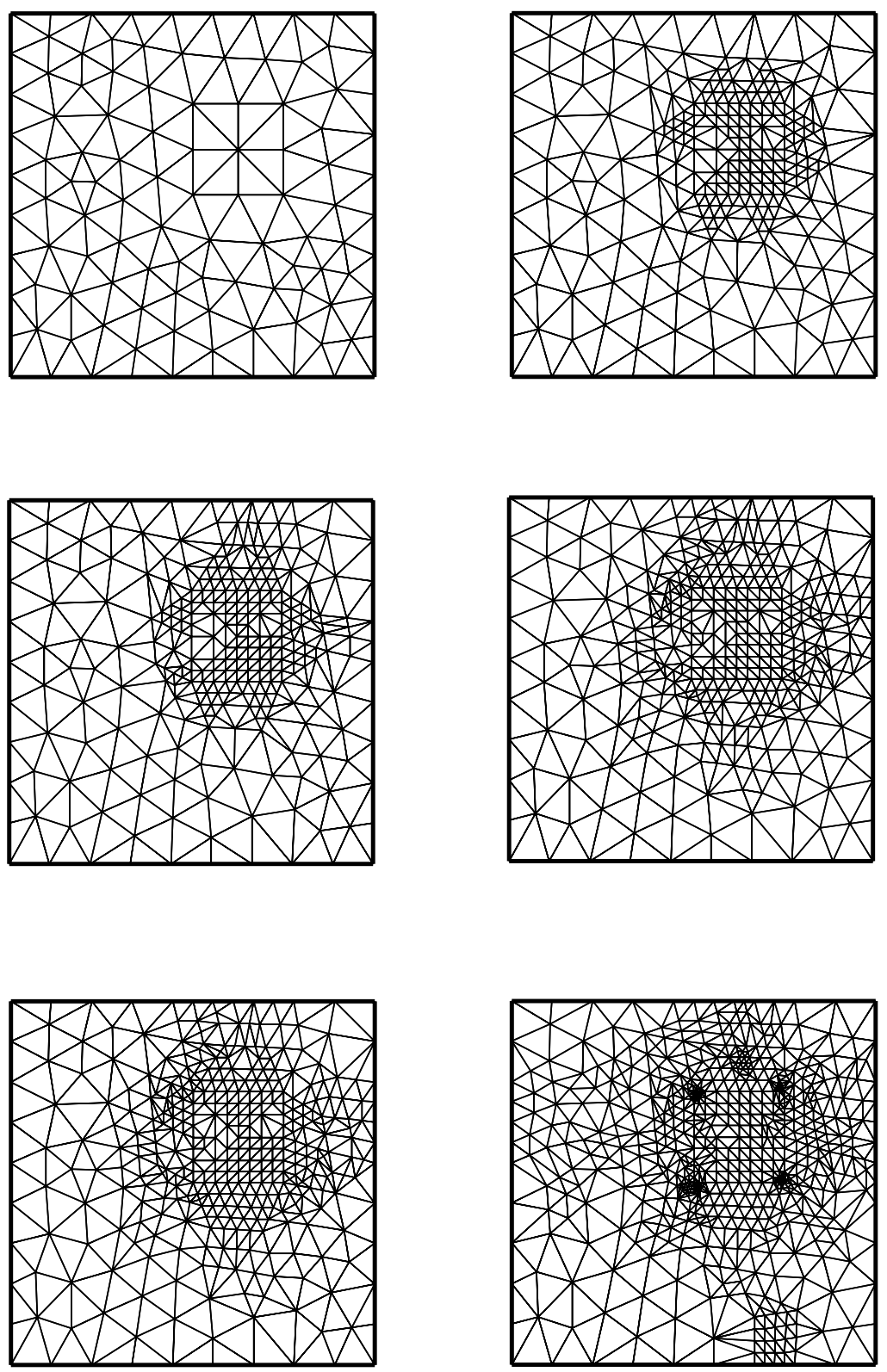

FiguRE 9.

The curves of isovalues of the discrete solution computed on the triangulation $\mathcal{T}_{h}^{5}$ are presented in Figure 10 for the mortar case. Indeed, their analogues in the conforming case are exactly the same.

The dimension of the final spaces $\mathbb{X}_{\delta}$ associated with $\mathcal{T}_{h}^{5}$ are very close, even if the number of triangles is larger in the conforming case. The quantities $\eta_{\text {norm }}^{n}$ and the associated discrete solutions are very similar. It can be noted that the complexity of mortar adaptivity comes from the structure of the data while the 


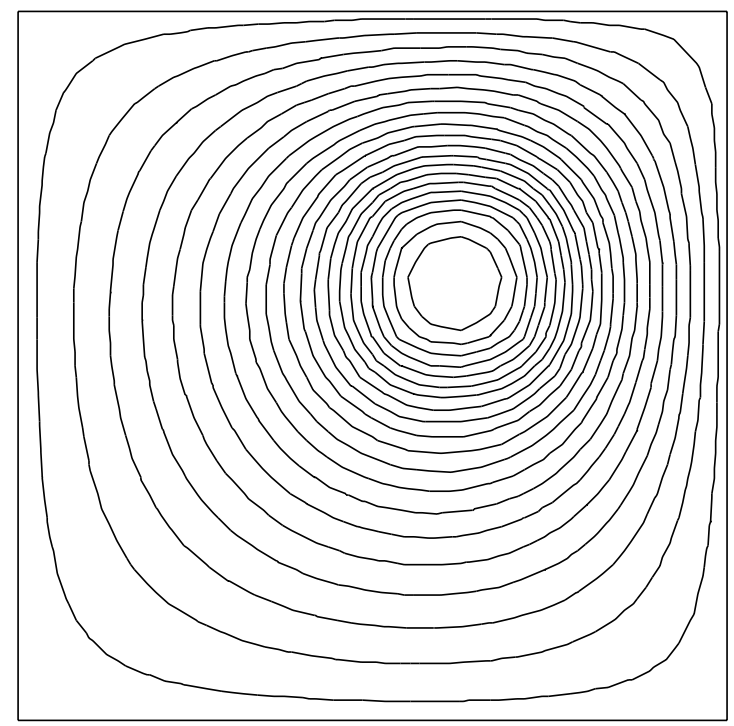

FigURE 10.

complexity of the conforming method is linked to the use of a mesh generator at each iteration.

We conclude with a slightly more difficult equation, where problem (1.1) is replaced by

$$
\begin{cases}-\operatorname{div}(a \operatorname{grad} u)=f & \text { in } \Omega, \\ u=0 & \text { on } \partial \Omega .\end{cases}
$$

Here, $\Omega$ is the square $]-1,1\left[^{2}\right.$, the coefficient $a$ is piecewise constant given by

$$
a(x, y)= \begin{cases}1 & \text { if } \left.(x, y) \in \Omega_{1}=\right]-1,0[\times]-1,1[ \\ 100 & \text { if } \left.(x, y) \in \Omega_{2}=\right] 0,1[\times]-1,1[\end{cases}
$$

and the data $f$ is equal to

$$
f(x, y)=\cos (\pi x) \cos (2 \pi y) .
$$

The definition of the $\eta_{e}$ remains unchanged. However, as explained in [9, Thm. 2.9] and in order that the constants in the a posteriori estimates do not depend on the variation of the function $a$, we replace the definition of the $\eta_{K}$ with

$$
\eta_{K}=h_{K} a_{K}^{-\frac{1}{2}}\left\|f_{\delta}+\operatorname{div}\left(a \operatorname{grad} u_{\delta}\right)\right\|_{L^{2}(K)}+\frac{1}{2} \sum_{e \in \mathcal{E}_{K}} h_{e}^{\frac{1}{2}} a_{e}^{-\frac{1}{2}}\left\|\left[a \partial_{\boldsymbol{n}} u_{\delta}\right]\right\|_{L^{2}(e)}
$$

where $a_{K}$ denotes the (constant) value of $a$ on $K$ and $a_{e}$ is equal to the value of $a$ on $e$ if $e$ is contained in $\Omega_{1}$ or $\Omega_{2}$, the largest value of $a$ if $e$ is contained in $\partial \Omega_{1} \cap \partial \Omega_{2}$. 

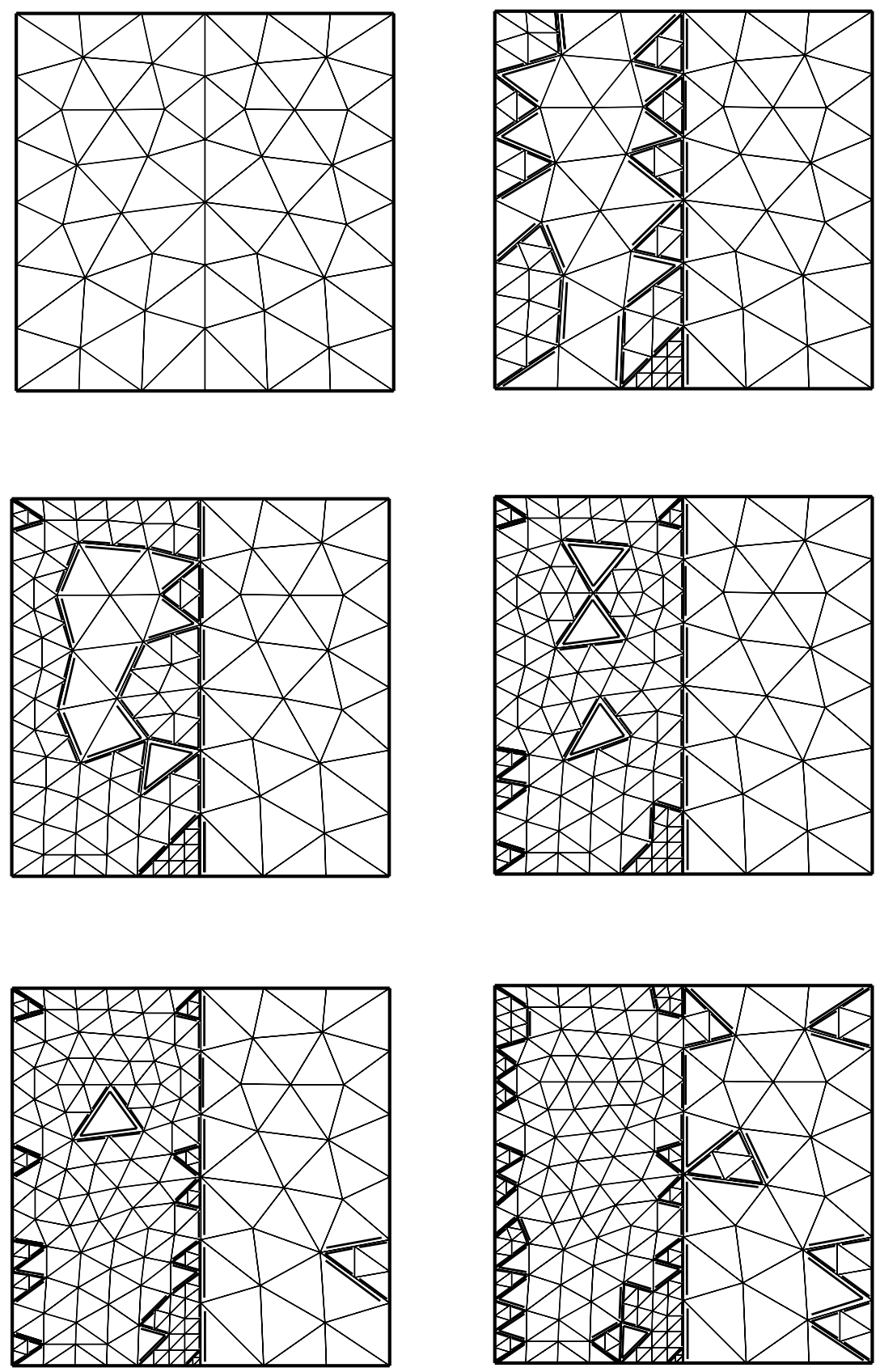

FiguRe 11.

Figure 11 represents the successive meshes $\mathcal{T}_{h}^{n}$, for $n=0, \ldots, 5$.

The same parameters as in the previous experiments, namely $N_{T}^{n}, M^{n}, \operatorname{dim} \mathbb{X}_{\delta}$ and $\eta_{\text {norm }}^{n}$, are given in Table 4. 
TABLE 4.

\begin{tabular}{|c|c|c|c|c|c|c|}
\hline$n$ & 0 & 1 & 2 & 3 & 4 & 5 \\
\hline$N_{T}^{n}$ & 84 & 141 & 186 & 231 & 261 & 306 \\
$M^{n}$ & 0 & 28 & 25 & 28 & 34 & 54 \\
$\operatorname{dim} \mathbb{X}_{\delta}$ & 145 & 308 & 386 & 480 & 554 & 675 \\
$\eta_{\text {norm }}^{n}$ & 0.2526 & 0.1290 & 0.0808 & 0.0601 & 0.0531 & 0.0466 \\
\hline
\end{tabular}

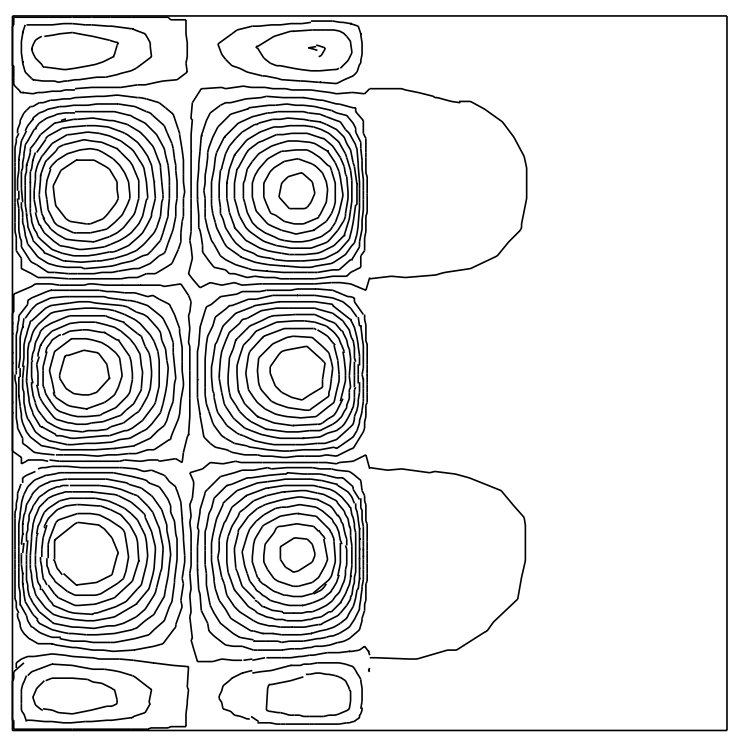

FIGURE 12.

Finally the curves of isovalues of the discrete solution computed on the triangulation $\mathcal{T}_{h}^{5}$ are presented in Figure 12.

\section{APPENDIX}

The aim of this Appendix is to prove the result stated in Proposition 3.1, namely to construct an operator $R_{\delta}$ from the space $\mathbb{X}^{n}$ introduced in Lemma 2.1 into the space $\mathbb{X}_{\delta}$ defined in (3.7) which satisfies the estimates, for all functions $v$ in $\mathbb{X}^{n}$

$$
\sum_{K \in \mathcal{T}_{h}^{n}}\left(h_{K}^{-2}\left\|v-R_{\delta} v\right\|_{L^{2}(K)}^{2}+\sum_{e \in \mathcal{E}_{K}} h_{e}^{-1}\left\|v-R_{\delta} v\right\|_{L^{2}(e)}^{2}\right) \leq c \mu_{\delta}\|v\|_{H_{\delta}^{1}(\Omega)}^{2} .
$$

We first recall from [13] (see also [4, Thm. 4.3]) the existence of an operator $R^{n, k}$ from the space of functions in $H^{1}\left(\Omega^{n, k}\right)$ vanishing on $\partial \Omega \cap \partial \Omega^{n, k}$ into the space $X_{-}^{n, k}$ of restrictions of functions of $\mathbb{X}_{\delta-}$ to $\Omega^{n, k}$ such that, for such a function $v$,

- the value of $R^{n, k} v$ at a corner $\boldsymbol{a}$ of a triangle $K$ in $\mathcal{T}_{h}^{n, k}$ that does not belong to $\partial \Omega$, is equal to the meanvalue of $v$ on the union $\bar{\Delta}_{a}^{k}$ of all triangles $K$ in $\mathcal{T}_{h}^{n, k}$ that contain $\boldsymbol{a}$, 


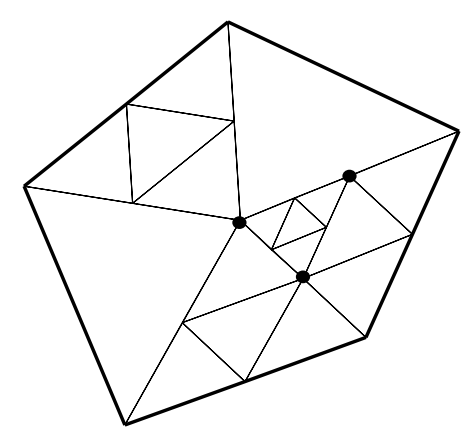

FiguRe 13.

- the following estimates hold for all $K$ in $\mathcal{T}_{h}^{n, k}$ and all $e$ in $\mathcal{E}_{K}$,

$$
\left\|v-R^{n, k} v\right\|_{L^{2}(K)} \leq c h_{K}\|v\|_{H^{1}\left(\Delta_{K}^{k}\right)}, \quad\left\|v-R^{n, k} v\right\|_{L^{2}(e)} \leq c h_{e}^{\frac{1}{2}}\|v\|_{H^{1}\left(\Delta_{e}^{k}\right)},
$$

where $\bar{\Delta}_{K}^{k}$, resp. $\bar{\Delta}_{e}^{k}$, stand for the union of the $\bar{\Delta}_{a}^{k}$ such that $\boldsymbol{a}$ is a corner of $K$, resp. an endpoint of $e$.

Moreover, the operator $R^{n, k}$ is continuous from $H^{1}\left(\Delta_{K}^{k}\right)$ into $H^{1}(K)$ with norm bounded independently of $\delta$. So the next estimate is easily derived by an interpolation argument (see [17, Chap. 1, Th. 5.1]): for $0<s<1$,

$$
\left\|v-R^{n, k} v\right\|_{H^{s}(K)} \leq c h_{K}^{1-s}\|v\|_{H^{1}\left(\Delta_{K}^{k}\right)} .
$$

The operator $R_{\delta}$ is built in four steps, more precisely we take

$$
R_{\delta}=R_{\delta}^{1}+R_{\delta}^{2}+R_{\delta}^{3}+R_{\delta}^{4} .
$$

We now describe the four steps successively.

Step 1. Local approximation. The operator $R_{\delta}^{1}$ is defined by

$$
\left(R_{\delta}^{1} v\right)_{\mid \Omega^{n, k}}=R^{n, k} v, \quad 0 \leq k \leq K^{n} .
$$

When multiplying the first estimate in (A.2) by $h_{K}^{-1}$ and summing its square on all $K$ in $\mathcal{T}_{h}^{n}$, we obtain the first part of (A.1) with $R_{\delta}$ replaced by $R_{\delta}^{1}$ (note that triangles contained in $\Delta_{K}^{k}$ appear at most a finite number of times in the sum, and that this number is bounded as a function of the regularity parameter $\sigma$ of the initial family of triangulations). Similarly, we prove the second part.

Step 2. Enforcing the continuity at common corners. Let $\tilde{\mathcal{V}}^{n, k}$ denote the set of all corners of triangles of $\mathcal{T}_{h}^{n, k}$ that belong to $\mathcal{S}^{n}$ and are also corners of triangles of another $\mathcal{T}_{h}^{n, k^{\prime}}$ (see Figure 13, where these corners are represented by black dots). So, each $\boldsymbol{a}$ in $\tilde{\mathcal{V}}^{n, k}$ belongs to several $\bar{\Omega}^{n, k^{\prime}}$, we denote by $k(\boldsymbol{a})$ the largest of such $k^{\prime}$. Denoting by $\varphi_{\boldsymbol{a}}$ the Lagrange function associated with $\boldsymbol{a}$, we set:

$$
\left(R_{\delta}^{2} v\right)_{\mid \Omega^{n, k}}=\sum_{\boldsymbol{a} \in \tilde{\mathcal{V}}^{n, k}}\left(\left(R^{n, k(\boldsymbol{a})} v\right)(\boldsymbol{a})-\left(R^{n, k} v\right)(\boldsymbol{a})\right) \varphi_{\boldsymbol{a} \mid \Omega^{n, k}}, \quad 0 \leq k \leq K^{n} .
$$



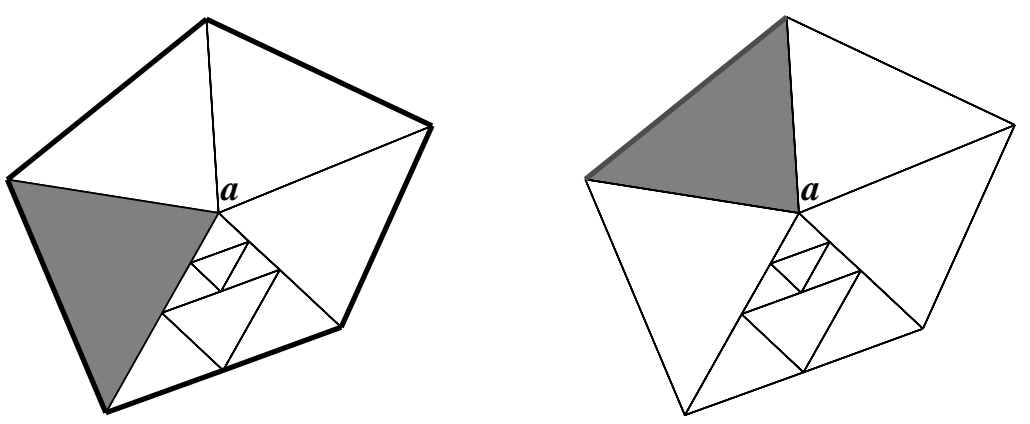

FIGURE 14.

Since the image of each $K$ in $\mathcal{T}_{h}^{n, k}$ by $\varphi_{\boldsymbol{a}}$ is contained in $[0,1]$, this yields for each $K$ and each $e$ in $\mathcal{E}_{K}$,

$$
\begin{gathered}
\left\|R_{\delta}^{2} v\right\|_{L^{2}(K)} \leq h_{K} \sum_{\boldsymbol{a} \in \tilde{\mathcal{V}}^{n, k} \cap K}\left|\left(R^{n, k(\boldsymbol{a})} v\right)(\boldsymbol{a})-\left(R^{n, k} v\right)(\boldsymbol{a})\right|, \\
\left\|R_{\delta}^{2} v\right\|_{L^{2}(e)} \leq h_{e}^{\frac{1}{2}} \sum_{\boldsymbol{a} \in \tilde{\mathcal{V}}^{n, k} \cap e}\left|\left(R^{n, k(\boldsymbol{a})} v\right)(\boldsymbol{a})-\left(R^{n, k} v\right)(\boldsymbol{a})\right| .
\end{gathered}
$$

So, we must now bound the quantities $\left|\left(R^{n, k(\boldsymbol{a})} v\right)(\boldsymbol{a})-\left(R^{n, k} v\right)(\boldsymbol{a})\right|$. As described in Figure 14 (where the $\Delta_{\boldsymbol{a}}^{k}$ are coloured in grey), two situations may occur:

- When $\Delta_{\boldsymbol{a}}^{k}$ and $\Delta_{\boldsymbol{a}}^{k(\boldsymbol{a})}$ are adjacent (where the term "adjacent" means that they share at least an edge $e$ of a triangle of $\mathcal{T}_{h}^{n, k(\boldsymbol{a})}$ containing $\left.\boldsymbol{a}\right)$, we introduce a continuous one-to-one mapping $F$ from the union $\bar{\Delta}_{\boldsymbol{a}}^{k} \cup \bar{\Delta}_{\boldsymbol{a}}^{k(\boldsymbol{a})}$ onto a reference subdomain $\hat{\Delta}$, which is affine on each triangle of $\mathcal{T}_{h}^{n}$ contained in $\bar{\Delta}_{\boldsymbol{a}}^{k} \cup \bar{\Delta}_{\boldsymbol{a}}^{k(\boldsymbol{a})}$ and maps the edge $e$ onto an edge $\hat{e}$ with length 1 , the edge $e^{\prime}$ of $\bar{\Delta}_{\boldsymbol{a}}^{k}$ that contains $e$ onto an edge $\hat{e}^{\prime}$. Then, if $\hat{w}$ stands for the function $w \circ F^{-1}$ for all functions $w$, we have

$$
\begin{aligned}
& \left|\left(R^{n, k(\boldsymbol{a})} v\right)(\boldsymbol{a})-\left(R^{n, k} v\right)(\boldsymbol{a})\right| \\
& \quad \leq\left\|\widehat{R^{n, k(\boldsymbol{a})}} v-\widehat{R^{n, k} v}\right\|_{L^{\infty}(\hat{e})} \\
& \quad \leq \hat{c}\left\|\widehat{R^{n, k(\boldsymbol{a})}} v-\widehat{R^{n, k} v}\right\|_{L^{2}(\hat{e})} \\
& \quad \leq \hat{c}\left(\left\|\hat{v}-\widehat{R^{n, k(\boldsymbol{a})}} v\right\|_{L^{2}(\hat{e})}+\left\|\hat{v}-\widehat{R^{n, k} v}\right\|_{L^{2}\left(\hat{e}^{\prime}\right)}\right) \\
& \quad \leq c\left(|v|_{H^{1}\left(\Delta_{e}^{k(\boldsymbol{a})}\right)}+|v|_{H^{1}\left(\Delta_{e^{\prime}}^{k}\right)}\right)
\end{aligned}
$$

which concludes the proof.

- When $\Delta_{\boldsymbol{a}}^{k}$ and $\Delta_{\boldsymbol{a}}^{k(\boldsymbol{a})}$ are not adjacent, there exists a finite number of triangles $K_{1}, \ldots, K_{r}$, such that $K_{1}$ is adjacent to $\bar{\Delta}_{\boldsymbol{a}}^{k(\boldsymbol{a})}, K_{r}$ is adjacent to $\bar{\Delta}_{\boldsymbol{a}}^{k}$ and each $K_{i}, 1 \leq i \leq r-1$, is adjacent to $K_{i+1}$ (the number of such triangles is bounded as a function of $\sigma$ ). Then, we write

$$
\begin{aligned}
& \left|\left(R^{n, k(\boldsymbol{a})} v\right)(\boldsymbol{a})-\left(R^{n, k}\right) v(\boldsymbol{a})\right| \\
& \quad \leq\left|\left(R^{n, k(\boldsymbol{a})} v\right)(\boldsymbol{a})-\bar{v}_{K_{1}}\right|+\left|\bar{v}_{K_{1}}-\bar{v}_{K_{2}}\right|+\cdots+\left|\bar{v}_{K_{r}}-\left(R^{n, k} v\right)(\boldsymbol{a})\right|,
\end{aligned}
$$


where each $\bar{v}_{K_{i}}$ stands for the meanvalue of $v$ on $K_{i}$, and we apply the same arguments as previously to each of these differences.

Noting that the support of $\varphi_{\boldsymbol{a}}$ is a finite number of triangles, bounded as a function of $\sigma$, we obtain (A.1) with $R_{\delta}$ raplaced by $R_{\delta}^{1}+R_{\delta}^{2}$.

Step 3. Enforcing the continuity at corners inside mortars. Exactly as for the proof of Lemma 2.3, with the same notation for $\mathcal{V}^{n, k}$, we take

$$
\left(R_{\delta}^{3} v\right)_{\mid \Omega^{n, k}}=\sum_{\boldsymbol{a} \in \mathcal{V}^{n, k}}\left[R_{\delta}^{1} v+R_{\delta}^{2} v\right](\boldsymbol{a}) \varphi_{\boldsymbol{a}}, \quad 0 \leq k \leq K^{n},
$$

where, if $\Omega^{n, k}$ coincides with $\Omega^{n, k_{i}(m)}$, the jump $\left[R_{\delta}^{1} v+R_{\delta}^{2} v\right](\boldsymbol{a})$ means

$$
\left(R_{\delta}^{1} v+R_{\delta}^{2} v\right)_{\mid \Omega^{n, k(m)}}-\left(R_{\delta}^{1} v+R_{\delta}^{2} v\right)_{\mid \Omega^{n, k_{i}(m)}} .
$$

The same arguments as in the proof of Lemma 2.3 allow for proving that (A.1) holds with $R_{\delta}$ raplaced by $R_{\delta}^{1}+R_{\delta}^{2}+R_{\delta}^{3}$.

Step 4. Enforcing the continuity through mortars. As in the proof of Lemma 2.3 and for the same operators $L_{i j}^{m}$ (restricted to the case $\ell=1$ of piecewise affine functions), the final idea consists in defining $R_{\delta}^{4}$ by

$$
R_{\delta}^{4} v=\sum_{m=1}^{M^{n}} \sum_{i=1}^{p(m)} \sum_{j=1}^{q(i)} R_{i j}^{m} v
$$

with

$$
R_{i j}^{m} v=L_{i j}^{m}\left(\left(R_{\delta}^{1} v+R_{\delta}^{2} v+R_{\delta}^{3} v\right)_{\mid \Omega^{n, k(m)}}-\left(R_{\delta}^{1} v+R_{\delta}^{2} v+R_{\delta}^{3} v\right)_{\mid \Omega^{n, k_{i}(m)}}\right) .
$$

Here, we use a slightly different estimate for the $L_{i j}^{m}$, which can be derived by exactly the same arguments as in [4, Thm. 5.1]: with the same notation as in Section 2, for $\frac{1}{2}<s<1$,

$$
\left\|L_{i j}^{m} \varphi\right\|_{H^{s}\left(\Delta_{j}^{k_{i}(m)}\right)} \leq c \mu_{\delta}^{s-\frac{1}{2}}|\varphi|_{H^{s-\frac{1}{2}}\left(\gamma_{i j}^{m}\right)} .
$$

It can also be checked by going to a reference element that

$$
\sum_{K \subset \Delta_{j}^{k_{i}(m)}} h_{K}^{-2}\left\|R_{i j}^{m} v\right\|_{L^{2}(K)}^{2} \leq c \sum_{K \subset \Delta_{j}^{k_{i}(m)}} h_{K}^{2 s-2}\left\|R_{i j}^{m} v\right\|_{H^{s}(K)}^{2} .
$$

So, using (A.5) yields

$$
\begin{aligned}
& \sum_{K \subset \Delta_{j}^{k_{i}(m)}} h_{K}^{-2}\left\|R_{i j}^{m} v\right\|_{L^{2}(K)}^{2} \\
& \quad \leq c\left(\sup _{K \subset \Delta_{j}^{k_{i}(m)}} h_{K}^{2 s-2}\right)\left\|R_{i j}^{m} v\right\|_{H^{s}\left(\Delta_{j}^{k_{i}(m)}\right)}^{2} \\
& \leq c \mu_{\delta}^{2 s-1}\left(\sup _{K \subset \Delta_{j}^{k_{i}(m)}} h_{K}^{2 s-2}\right) \\
& \quad \times\left\|\left(R_{\delta}^{1} v+R_{\delta}^{2} v+R_{\delta}^{3} v\right)_{\mid \Omega^{n, k(m)}}-\left(R_{\delta}^{1} v+R_{\delta}^{2} v+R_{\delta}^{3} v\right)_{\mid \Omega^{n, k_{i}(m)}}\right\|_{H^{s-\frac{1}{2}\left(\gamma_{i j}^{m}\right)}}^{2} .
\end{aligned}
$$


Adding and subtracting $v$ in the previous difference and using the trace theorem (obtained by going to a reference element), we derive

$$
\begin{aligned}
& \sum_{K \subset \Delta_{j}^{k_{i}(m)}} h_{K}^{-2}\left\|R_{i j}^{m} v\right\|_{L^{2}(K)}^{2} \\
& \leq c \mu_{\delta}^{2 s-1}\left(\sup _{K \subset \Delta_{j}^{k_{i}(m)}} h_{K}^{2 s-2}\right) \\
& \quad \times\left(\left\|v-\left(R_{\delta}^{1} v+R_{\delta}^{2} v+R_{\delta}^{3} v\right)\right\|_{H^{s}\left(\tilde{\Delta}_{j}^{k_{i}(m)}\right)}^{2}\right. \\
& \left.\quad+\left\|v-\left(R_{\delta}^{1} v+R_{\delta}^{2} v+R_{\delta}^{3} v\right)\right\|_{H^{s}\left(\tilde{\Delta}_{j}^{k_{i}(m)}\right)}^{2}\right) .
\end{aligned}
$$

Next, we derive from (A.3) and the fact that the norm of each function $\varphi_{\boldsymbol{a}}$ in $H^{s}(K)$ is bounded by $h_{K}^{1-s}$ that, for all $K$ of $\mathcal{T}_{h}^{n, k}$,

$$
\left\|v-\left(R_{\delta}^{1} v+R_{\delta}^{2} v+R_{\delta}^{3} v\right)\right\|_{H^{s}(K)} \leq h_{K}^{1-s}\|v\|_{H^{1}\left(\Delta_{K}^{k}\right)} .
$$

Since all triangles in $\Delta_{j}^{k_{i}(m)}$ have the same diameter, we obtain

$$
\begin{aligned}
& \sum_{K \subset \Delta_{j}^{k_{i}(m)}} h_{K}^{-2}\left\|R_{i j}^{m} v\right\|_{L^{2}(K)}^{2} \\
& \quad \leq c \mu_{\delta}^{2 s-1}\left(\sup _{K \subset \Delta_{j}^{k_{i}(m)}} h_{K}^{2 s-2}\right)\left\|v-\left(R_{\delta}^{1} v+R_{\delta}^{2} v+R_{\delta}^{3} v\right)\right\|_{H^{s}\left(\tilde{\Delta}_{j}^{k_{i}(m)}\right)}^{2} \\
& \quad+c^{\prime} \mu_{\delta}^{2 s-1}\|v\|_{H^{1}\left(\Delta_{j *}^{k_{i}(m)}\right)}^{2},
\end{aligned}
$$

where $\Delta_{j *}^{k_{i}(m)}$ is a neighbourhood of $\Delta_{j}^{k_{i}(m)}$ in $\Omega^{n, k_{i}(m)}$. Finally, we sum up the previous inequality on the $j, i$ and $m$ and note that the diameter of the triangles contained in $\Delta_{j}^{k_{i}(m)}$ are smaller than $\mu_{\delta}$ times the diameter of the triangle of $\mathcal{T}_{h}^{n, k(m)}$ that contains $\tilde{\Delta}_{j}^{k_{i}(m)}$, so that

$$
\begin{aligned}
& \sum_{K \in \mathcal{T}_{h}^{n}} h_{K}^{-2}\left\|R_{\delta}^{4} v\right\|_{L^{2}(K)}^{2} \\
& \quad \leq c \mu_{\delta} \sum_{K \in \mathcal{T}_{h}^{n}} h_{K}^{2 s-2}\left\|v-\left(R_{\delta}^{1} v+R_{\delta}^{2} v+R_{\delta}^{3} v\right)\right\|_{H^{s}(K)}^{2}+c^{\prime} \mu_{\delta}^{s-\frac{1}{2}}\|v\|_{H_{\delta}^{1}(\Omega)}^{2} .
\end{aligned}
$$

Using (A.6) once more yields the desired result. The second estimate in (A.1) is obtained by similar arguments.

It is now readily checked that the operator $R_{\delta}$ defined in (A.4) takes its values into $\mathbb{X}_{\delta-}$ and that estimates (A.1) are satisfied, which concludes the proof.

\section{ACKNOWLEDGMENTS}

The authors are deeply grateful to an anonymous referee, whose very interesting and helpful comments greatly improved this work.

\section{REFERENCES}

[1] M. Azaïez, C. Bernardi, Y. Maday - Some tools for adaptivity in the spectral element method, in Proc. of the third Int. Conf. On Spectral And High Order Methods, Houston J. of Math. (1996), 243-253.

[2] F. Ben Belgacem - The Mortar finite element method with Lagrange multipliers, Numer. Math. 84 (1999), 173-197. MR 2001b:65101 
[3] F. Ben Belgacem, C. Bernardi, N. Chorfi, Y. Maday - Inf-sup conditions for the mortar spectral element discretization of the Stokes problem, Numer. Math. 85 (2000), 257-281.CMP 2000:12

[4] C. Bernardi, V. Girault - A local regularization operator for triangular and quadrilateral finite elements, SIAM J. Numer. Anal. 35 (1998), 1893-1916. MR 99g:65107

[5] C. Bernardi, Y. Maday - Mesh adaptivity in finite elements by the mortar method, Revue européenne des éléments finis 9 (2000), 451-465.

[6] C. Bernardi, Y. Maday, A.T. Patera - A new nonconforming approach to domain decomposition : the mortar element method, Collège de France Seminar XI, H. Brezis \& J.-L. Lions eds., Pitman (1994), 13-51. MR 95a:65201

[7] C. Bernardi, Y. Maday, A.T. Patera - Domain decomposition by the mortar element method, in Asymptotic and Numerical Methods for Partial Differential Equations with Critical Parameters, H.G. Kaper \& M. Garbey eds., N.A.T.O. ASI Series C 384 (1993), 269-286. MR 94c:65151

[8] C. Bernardi, R.G. Owens, J. Valenciano - An error indicator for mortar element solutions to the Stokes problem, Internal Report 99030, Laboratoire d'Analyse Numérique, Université Pierre et Marie Curie, Paris (1999).

[9] C. Bernardi, R. Verfürth - Adaptive finite element methods for elliptic equations with nonsmooth coefficients, Numer. Math. 85 (2000), 579-608. MR 2001e:65177

[10] D. Braess, R. Verfürth - A posteriori error estimators for the Raviart-Thomas element, SIAM J. Numer. Anal. 33 (1996), 2431-2444. MR 97m:65201

[11] F. Bouillault, A. Buffa, Y. Maday, F. Rapetti - The mortar edge element method in three dimensions: application to magnetostatics, Internal Report, Laboratoire d'Analyse Numérique, Université Pierre et Marie Curie, Paris (2000).

[12] P.G. Ciarlet - Basic Error Estimates for Elliptic Problems, in the Handbook of Numerical Analysis, Vol. II, P.G. Ciarlet \& J.-L. Lions eds., North-Holland (1991), 17-351. CMP 91:14

[13] P. Clément - Approximation by finite element functions using local regularization, R.A.I.R.O. Anal. Numér. 9 (1975), 77-84. MR 53:4569

[14] M. Crouzeix - Personal communication.

[15] F. Hecht, O. Pironneau - Multiple meshes and the implementation of freefem+, I.N.R.I.A. Report, Rocquencourt (1999).

[16] P. Joly - Remise en forme, analyse numérique matricielle, Cours de D.E.A., Laboratoire d'Analyse Numérique, Université Pierre et Marie Curie, Paris (1999).

[17] J.-L. Lions, E. Magenes - Problèmes aux limites non homogènes et applications, Vol. 1, Dunod (1968). MR 40:512

[18] L.R. Scott, S. Zhang - Finite element interpolation of nonsmooth functions satisfying boundary conditions, Math. Comput. 54 (1990), 483-493. MR 90j:65021

[19] R. Verfürth - A posteriori error estimators for the Stokes equations, II non-conforming discretizations, Numer. Math. 60 (1991), 235-249. MR 92j:65189

[20] R. Verfürth - A Review of A Posteriori Error Estimation and Adaptive Mesh-Refinement Techniques, Wiley \& Teubner (1996).

[21] R. Verfürth - Error estimates for some quasi-interpolation operators, Modél. Math. et Anal. Numér. 33 (1999), 695-713.

[22] O.B. Widlund - An extension theorem for finite element spaces with three applications, in Numerical Techniques in Continuum Mechanics, Proceedings of the Second GAMM Seminar, W. Hackbush \& K. Witsch eds., Kiel (1986).

[23] B. Wohlmuth - A residual based error estimator for mortar finite element discretizations, Numer. Math. 84 (1999), 143-171. MR 2000h:65155

Analyse Numérique, C.N.R.S. et Université Pierre et Marie Curie, B.C. 187, 4 Place Jussieu, 75252 Paris Cedex 05, France

E-mail address: bernardi@ann.jussieu.fr

Analyse Numérique, C.N.R.S. et Université Pierre et Marie Curie, B.C. 187, 4 Place Jussieu, 75252 Paris Cedex 05, France

E-mail address: hecht@ann.jussieu.fr 